Mon. Not. R. Astron. Soc. 000,1-?? (2005) Printed 29 October $2018 \quad$ (MN LATEX style file v2.2)

\title{
Detecting quasars at very high redshift with next generation $\mathrm{X}$-ray telescopes
}

\author{
Kirsty J. Rhook \& Martin G. Haehnelt† \\ Institute of Astronomy, Madingley Road, Cambridge CBз OHA
}

Revised Version May 13th, 2008

\begin{abstract}
The next generation of X-ray telescopes have the potential to detect faint quasars at very high redshift and probe the early growth of massive black holes (BHs). We present modelling of the evolution of the optical and X-ray AGN luminosity function at $2<z<6$ based on a CDM merger-driven model for the triggering of nuclear activity combined with a variety of fading laws. We extrapolate the merger-driven models to $z>6$ for a range of $\mathrm{BH}$ growth scenarios. We predict significant numbers of sources at $z \sim 6$ with fluxes just an order of magnitude below the current detection limits and thus detectable with XEUS and Constellation-X, relatively independently of the fading law chosen. The predicted number of sources at even higher redshift depends sensitively on the early growth history of BHs. For passive evolution models in which BHs grow constantly at their Eddington limit, detectable BHs may be rare beyond $z \sim 10$ even with Generation-X. However, in the more probable scenario that $\mathrm{BH}$ growth at $z>6$ can be described by passive evolution with a small duty cycle, or by our merger driven accretion model, then we predict that XEUS and Generation-X will detect significant numbers of black holes out to $z \sim 10$ and perhaps beyond.
\end{abstract}

Key words: quasars: general - cosmology: theory

\section{INTRODUCTION}

- The next generation of X-ray satellites promise to be powerful probes of quasar activity out to very high redshifts. The European lead project XEUS1 is designed to reach a sensitivity $\sim 100$ better than the deepest observations to date. Constellation- $\mathrm{X}^{2}$ would offer at least an order of magnitude improvement in sensitivity, hopefully paving the way for a mission like Generation- $\mathrm{X}^{3}$ which could probe 50 times fainter again.

In this paper we aim to assess the prospects for probing the early growth of supermassive black holes (SMBHs) at $z \gtrsim 6$ with these telescopes or similar future X-ray missions [see, e.g., Haiman \& Loeb (1999) and Wyithe \& Loeb (2003) for earlier work on this]. The observed correlations between SMBH mass and galaxy properties, which are now well established (e.g. Gebhardt et al. 2000; Ferrarese \& Merritt 2000), strongly suggest a tight link between the build-up of the stellar mass in galactic bulges and the mass of the central BH. Most mod-

\footnotetext{
* krhook@ast.cam.ac.uk

† haehnelt@ast.cam.ac.uk

1 http://www.rssd.esa.int/index.php?project=XEUS

2 http://constellation.gsfc.nasa.gov/

3 htt://www.cfa.harvard.edu/hea/genx.html
}

els of the growth history of SMBHs assume that the frequent merging of galaxies predicted by CDM-like hierarchical models of galaxy formation plays an important role in this process (e.g. Kauffmann \& Haehnelt 2000; Wyithe \& Loeb 2003; Volonteri et al. 2003; Croton et al. 2006; Malbon et al. 2006). Such models, although subject to considerable degeneracies, have been reasonably successful in reproducing the observed luminosity function of AGN as well as the inferred $\mathrm{BH}$ mass function and their respective evolution with redshift. These models generally struggle, however, to combine the very efficient high redshift growth of BHs, suggested by the SMBHs detected at $z \sim 6$, with the strong feedback required to reproduce the rapid decline in the fuelling rate of the most massive BHs at low redshift (Bromley et al. 2004; Malbon et al. 2006). Furthermore, at low redshift there is considerable observational evidence that some, and maybe even most, of the fuelling of SMBHs is not connected to major mergers between galaxies (e.g. Kauffmann et al. 2003; Li et al. 2007a). This may be related to the fact that merger-driven models of quasar activity have difficulty reproducing the luminosity dependent density evolution (LDDE) observed for faint AGN at redshifts below $z \sim 3$ (e.g. Ueda et al. 2003; Hasinger et al. 2005; Silverman et al. 2007; Bongiorno et al. 2007) [see, e.g., Marulli et al. (2007)], although more sophisticated models which follow the simultaneous growth of galaxies and AGN 
can incorporate feedback in such a way to orchestrate this effect (Fontanot et al. 2006).

We explore predictions for a variety of models for the early growth of SMBHs which range from merger driven accretion to continuous Eddington-limited accretion. The $\mathrm{BH}$ growth scenario is crucial in determining the number of active BHs that we expect to detect as faint X-ray sources. However, as X-ray observations push to fainter flux limits they are expected to discover a mixture of low-luminosity objects at low to intermediate redshift, as well as distant objects previously at undetectable redshifts. Predictions for the redshift distribution of faint sources, which encrypt the growth history of SMBHs, therefore require a simultaneous study of the faint end of the luminosity function at all redshifts.

We have adopted a hybrid approach to modelling the redshift evolution of the X-ray emission associated with the fuelling of SMBHs. At low redshift $(z \lesssim 2)$ we use the observed X-ray luminosity functions, which are now well established down to very faint luminosities. At intermediate redshifts the faint end of the X-ray luminosity function is still subject to some uncertainty due to the difficultly of assigning redshifts to faint sources (Aird et al. 2008). At $2<z<6$ we have therefore adopted a CDM merger-driven model for the evolution of the emission from SMBHs, constrained by the available optical and X-ray data. The efficiency of BH formation, the quasar lifetime and accretion rate, and the quasar spectral energy distribution (SED) are all free parameters in such a model. The relevant assumptions should obviously be guided by our empirical and physical understanding of quasars, but there is still much flexibility, particularly near the observational limits in redshift and luminosity.

Observationally, faint AGN are more likely to display signatures of obscuration by gas and/or dust than brighter sources (Ueda et al. 2003; Simpson 2005; Treister et al. 2006; Maiolino et al. 2007). Most semi-analytic models of quasar activity do not model obscuration effects in sufficient detail to be able to explain these trends. Hopkins et al. 2005a b, 2006, 2007) have, however, recently presented a model for the quasar luminosity function (QLF) at $z<5$, which includes prescriptions for quasar luminosity dependent fading and absorption, to successfully reconcile the optical and X-ray QLFs. We follow a very similar approach and adopt many of the same assumptions. However, whereas Hopkins et al. (2006) extract the quasar formation rate from the constraints provided by the observed QLF, we use a cosmological galaxy merger rate from the extended PressSchechter formalism (e.g. Lacey \& Cole 1993). This will allow us to extend our model to the redshifts $(z>6)$ we are predominantly concerned with in a well-motivated way. Semi-analytic models are an efficient way of exploring the wide range of physically plausible evolutionary scenarios for the QLF. As we will discuss in some detail, the faint end of the luminosity functions, and therefore the redshift distribution of faint X-ray sources, is very sensitive to the assumed time dependence of the fading of the emission in different wave-bands during galaxy mergers. We utilise the flexibility of our model to study the constraints on this fading rate provided by the optical, soft and hard X-ray QLFs, and the unresolved Cosmic X-ray background (CXRB).

We then go on to explore several models for $\mathrm{BH}$ growth at $z>6$ which are consistent with the observed data at $0<z<6$ and span a wide range of possibilities for the rate of $\mathrm{BH}$ growth at high redshift.

Our paper is structured as follows. In Section 2 we briefly review the constraints on the optical and X-ray QLFs. We describe our merger driven model for $0<z<6$ in Section 3, and the high redshift extensions in Section 4.3. In the main body of Section 4 we calibrate our model against the measured optical and X-ray QLFs. In Section 5 we examine the consistency of our models with the CXRB and observed $\log \mathrm{N}-\log \mathrm{S} \mathrm{X}$-ray source distribution, and present our prediction for the source density detectable with future missions, before concluding in Section 6 .

Throughout this paper we adopt a cosmological matter density $\Omega_{m}=0.27$, baryonic matter density $\Omega_{b}=0.044$, cosmological constant $\Omega_{\Lambda}=0.73$, present day Hubble constant $H_{o} \equiv 100 h \mathrm{~km} \mathrm{~s}^{-1} \mathrm{Mpc}^{-1}=71 \mathrm{~km} \mathrm{~s}^{-1} \mathrm{Mpc}^{-1}$, a mass variance on scales of $8 h^{-1} \mathrm{Mpc} \sigma_{8}=0.84$ and a scale invariant primordial power spectrum (slope $n=1$ ).

\section{OBSERVATIONAL CONSTRAINTS}

We are primarily interested in exploring the constraints on the early growth of SMBHs that may be provided by future $\mathrm{X}$-ray missions. In order to do this in a conservative way we tie our model to a wide range of observational constraints on the growth history of SMBHs at low and intermediate redshift offered by the QLF in the optical, soft and hard $\mathrm{X}$-ray bands, as well as the CXRB.

The luminosity function of optically selected QSOs is well measured up to $z \gtrsim 4$, with constraints on the bright end that reach up to $z \sim 6$ (Croom et al. 2004; Wolf et al. 2003; Fan et al. 2003, 2004; Jiang et al. 2007). The constraints on the X-ray QLF extend to $z \sim 4$ at best, but deep $\mathrm{X}$-ray surveys surveys reach to considerably fainter limiting magnitudes than optical surveys. Combined constraints of wide-field and deep surveys result in a dynamic range of $\sim 5$ orders of magnitude in luminosity at $z \sim 2$ compared to $\sim 3$ orders of magnitude at optical wavelengths. The low luminosities reached by deep X-ray surveys has allowed Shankar \& Mathur (2007) to constrain the faint-end slope of the optical QLF at $z \sim 6$ from the dearth of X-ray detections above $z \sim 4-5$.

The density of optically bright quasars peaks at $z \sim 3$ with quasar activity decreasing towards both lower and higher redshift (e.g. Boyle et al. 2000; Wolf et al. 2003; Croom et al. 2004; Richards et al. 2006). The optical QLF is well fit by a double power-law with the break luminosity evolving self-similarly with redshift, although there is evidence for the bright end slope flattening toward high redshift (Fan et al. 2004; Richards et al. 2006) [but see also Fontanot et al. (2007)]. More recent evidence that the space density of lower luminosities sources peaks at $1<z<1.5$ (Bongiorno et al. 2007) suggests that the full picture is probably more complicated, and that the faint optically selected sources show a similar evolution to that seen in X-ray surveys. The X-ray luminosity function is best fit with a luminosity dependent density evolution (e.g. Mivaji et al. 2001; Ueda et al. 2003; Fiore et al. 2003; Hasinger et al. 2005; Silverman et al. 2007) with the source density peaking at $z \sim 0.7-1$. Additional constraints at X-ray wavelengths come from the measurements of the CXRB (Worslev et al. 
2005; Hickox \& Markevitch 2006). Obviously the integrated emission from faint AGN below the current detection limits cannot exceed the measured unresolved CXRB and measurements of the background intensity are thus probably the strongest constraints on the faint end of the X-ray luminosity function.

\section{A HYBRID MODEL FOR THE EVOLUTION OF THE EMISSION DUE TO ACCRETION ONTO SUPERMASSIVE BLACK HOLES}

\subsection{Rationale for a hybrid model}

As motivated in the introduction, we have chosen a hybrid approach to modelling the evolution of the emission due to accretion onto SMBHs. At low redshift $(z<2)$ we use the well established observed X-ray luminosity functions, which extend to impressively faint luminosities. At intermediate redshift, where the X-ray luminosity function becomes less reliable, we use a merger-driven CDM-like model with a range of assumptions for the decline of the accretion rate onto the central BH during a merger. This model is calibrated using the extensive optical data as well as the available X-ray data. In Section 4 we describe an extension of the merger-driven model, as well as models for growth via continuous Eddington limited accretion with a range of duty cycles, to very high redshift $(z>6)$. Recently Shankar et al. (2007) constructed an observationally anchored model for the evolution of the SMBH population which indicates that the quasar duty cycle increases with increasing redshift, providing some empirical impetus for this approach.

We first discuss the ingredients of our merger-driven model in some detail.

\subsection{A CDM-like merger driven model for the luminosity function at intermediate redshift}

We assume that the accretion of gas onto SMBHs is predominantly triggered by major galaxy mergers (Kauffmann \& Haehnelt 2000; Di Matteo et al. 2005; Hopkins et al. 2005a), and take the merger rate of dark matter haloes in the standard $\Lambda C D M$ model for structure formation as a proxy for the rate of galaxy mergers. This picture is admittedly simple but has nevertheless been shown to yield a reasonably consistent model for many of the properties of the observed QLF and its evolution at intermediate redshifts (e.g. Haehnelt et al. 1998; Wvithe \& Loeb 2002, 2003; Volonteri et al. 2003; Croton et al. 2006; Marulli et al. 2007).

In a merger-driven model, new quasars continuously form at a redshift and luminosity dependent rate. We assume that the quasars become active with an initial peak luminosity $L_{\text {peak }}$ and then fade according to a "fading law", $\frac{d t}{d \log L}$. For a given model for the rate of formation of sources with peak luminosity $L_{\text {peak }}, \dot{n}\left(L_{\text {peak }}\right)=\frac{d^{2} n}{d \log L_{\text {peak }} d t}$, the luminosity function, $\frac{d n}{d \log L}$, can be written as

$$
\frac{d n}{d \log L}=\int_{L}^{\infty} \frac{d t}{d \log L}\left(L, L_{\text {peak }}\right) \frac{d^{2} n}{d \log L_{\text {peak }} d t} d \log L_{\text {peak }} .
$$

We assume that $L_{\text {peak }}$ is the Eddington luminosity of the final mass $\mathrm{BH}$. We further assume that dark matter halos host a central $\mathrm{BH}$ with mass $M_{\mathrm{bh}}$ given by (e.g. Wvithe \& Loeb 2003)

$$
\begin{aligned}
M_{\mathrm{bh}}= & \epsilon(M, z) M, \\
= & \epsilon_{o} h^{\alpha / 3}\left[\frac{\Omega_{m} \Delta_{c}}{\Omega_{m}^{z} 18 \pi^{2}}\right]^{\alpha / 6} \\
& \times(1+z)^{\alpha / 2}\left(\frac{M}{10^{12} M_{\odot}}\right)^{\alpha / 3-1} M,
\end{aligned}
$$

where $\Omega_{m}^{z}=\frac{\Omega_{m}(1+z)^{3}}{\Omega_{m}(1+z)^{3}+\Omega_{\Lambda}+\Omega_{k}(1+z)^{2}}, d \equiv \Omega_{m}^{z}-1$ and $\Delta_{c}=18 \pi^{2}+82 d-39 d^{2}$ is the overdensity of a virialised halo at redshift $z$. This relationship is motivated by the observed correlation between $M_{\mathrm{bh}}$ and the velocity dispersion of the host galaxie's bulge, $\sigma$ (Ferrarese 2002; Shields et al. 2003). We assume that $\sigma$ may be approximated by $v_{\text {vir }} / \sqrt{2}$, where $v_{\text {vir }}$ is the virial velocity of the dark matter halo from Barkana \& Loeb (2001). Empirical estimates of $\alpha$ typically fall in the range $4-5$. We have chosen $\alpha=5$ which is consistent with a simple self-regulated growth scenario in which the $\mathrm{BH}$ grows until it radiates enough energy to unbind the gas that is feeding it (e.g. Silk \& Rees 1998; Haehnelt et al. 1998; Wyithe \& Loeb 2003). The merger rate $\frac{d^{2} n}{d \log L_{\text {peak }} d t}$ is taken as the merger rate of halos with final BH mass such that $L_{\text {peak }}=L_{\mathrm{Edd}}\left(M_{\mathrm{bh}}\right)$. Using equation (2) to relate $\mathrm{BH}$ and host halo mass, we may write

$$
\frac{d^{2} n}{d \log L_{\text {peak }} d t}=\frac{3}{\alpha} M \int_{\Delta M=0.25(M-\Delta M)}^{0.5(M-\Delta M)} \dot{N}(M, \Delta M, t) d \Delta M
$$

where the limits of integration ensure that we count only major mergers and $\dot{N}(M, \Delta M, t)$ is the merger rate of dark matter haloes of mass $M-\Delta M$ and $\Delta M$ per unit cosmic time $t$,

$$
N(M, \Delta M, t)=\left.\frac{d^{2} P}{d \Delta M d t}\right|_{M-\Delta M} \frac{d n}{d(M-\Delta M)} .
$$

Here $\left.\frac{d^{2} P}{d \Delta M d t}\right|_{M-\Delta M}$ is the probability per unit time that a halo mass $\Delta M$ will merge with another halo to form a halo with mass $M$ from Lacev \& Cole (1993), and $\frac{d n}{d(M-\Delta M)}$ is the space density of halos with the appropriate mass difference from Press \& Schechter (1974) (with modification from Sheth \& Tormen (1999), see Rhook \& Haehnelt (2006) and Wvithe \& Loeb (2003) for similar calculations).

Equation (3) becomes inaccurate as $\frac{d t}{d \log L}$ approaches the Hubble time at the relevant redshift. This could in principle be relevant at high redshift, however at high redshift we are mainly concerned with the bright end of the QLF for which the fading time-scale remains short (see Section 3.31$)$. Note that for $\frac{d t}{d \log L}\left(L, L_{\text {peak }}\right)=$ constant (corresponding to an exponential light-curve) or $\frac{d t}{d \log L}\left(L, L_{\text {peak }}\right) \propto$ $\delta\left(L-L_{\text {Lpeak }}\right)$ (corresponding to a top-hat light-curve) the shape of the QLF is identical to that of $\dot{n}\left(L_{\text {peak }}\right)$.

\subsection{Gas accretion, quasar lifetime and fading laws, and the minimum black hole mass}

As discussed in the previous section, the faint end of the QLF is very sensitive to the assumed fading law. When 
two gas-rich galaxies merge, the resulting tidal torques drive large amounts of gas into the central region, providing fuel for the rapid growth of a SMBH. The fading of the quasar emission due the gas accretion onto a central SMBH is governed by the infall of gas to the centre and its subsequent accretion onto the SMBH during the late stages of the merger. Hopkins et al. (2005a) used numerical simulations of galaxy mergers, with a prescription for the subsequent accretion of gas onto a central SMBH from Di Matteo et al. (2005), to obtain a physically motivated fading law. Hopkins et al. (2005a) present a power-law representation for this fading law, $\frac{d t}{d \log L}$, which has been fit to the results of several hundred simulations of mergers between equal mass galaxies resulting in quasars with peak bolometric luminosities between $10^{8}$ and $10^{15} \mathrm{~L} \odot$. The power-law index depends on $L_{\text {peak }}$, with higher luminosity quasars expected to spend relatively more time close to their Eddington limit,

$$
\begin{aligned}
\frac{d t\left(L, L_{\text {peak }}\right)}{d \log L} & =\left|\alpha_{L}\right| t_{9}\left(\frac{L}{10^{9} \mathrm{~L}_{\odot}}\right)^{\alpha_{L}\left(L_{\text {peak }}\right)} \\
\alpha_{L}\left(L_{\text {peak }}\right) & =-0.95+0.32 \log _{10}\left(L_{\text {peak }} / 10^{12} \mathrm{~L}_{\odot}\right)
\end{aligned}
$$

where $t_{9} \approx 10^{9}$ yrs and $\alpha_{L} \geqslant-0.2$. Hopkins et al. (2005a) model the quasar luminosity over the entire merger process and therefore equation (5) includes the activity of the quasar prior to the luminosity reaching $L_{\text {peak }}$. This distinction should not be important for our modelling.

In order to investigate the effect of the quasar lightcurve on the the faint end of the luminosity function, we consider the fading law as proposed by Hopkins et al. (2005a) as well as a fading law where quasars fade more rapidly and spend equal amounts of time at each luminosity (relative to the peak luminosity) independently of $L_{\text {peak }}$,

$$
\frac{d t}{d \log L}=\left|\alpha_{L}\right| t_{q}\left(\frac{L}{L_{\text {peak }}}\right)^{\alpha_{L}} .
$$

Here $\alpha_{L}$ is a non-positive constant and larger values of $\left|\alpha_{L}\right|$ result in light curves for which quasars spend relatively more time at sub-Eddington luminosities. Note that values of $\alpha_{L}$ that are much less than zero are excluded by the observational data.

The faint end of the luminosity function is also sensitive to the minimum mass of a $\mathrm{BH}$ powering a quasar, $M_{\mathrm{bh}, \min }$, which for Eddington limited growth is equivalent to the minimum peak luminosity of a quasar, $L_{\text {peak,min }}$. There are very few claimed detections of SMBHs with masses smaller than $10^{6} \mathrm{M}_{\odot}$ (but see Greene \& Ho 2007a), and Greene \& Ho (2007b) find that the mass function of local low-luminosity active BHs turns over at $M_{\mathrm{bh}} \sim 10^{6} \mathrm{M}_{\odot}$. It is also a matter of intense debate whether the build-up of SMBHs by hierarchical merging extends to intermediate mass BHs in the mass range $100 \mathrm{M}_{\odot}-10^{6} \mathrm{M}_{\odot}$ (e.g. Haehnelt 2004; Volonteri et al. 2007). We therefore also explore the effect of varying the minimum $\mathrm{BH}$ mass, $M_{\mathrm{bh}, \min }$, in our models.

\subsection{Modelling the Spectral Energy Distribution}

To relate the intrinsic bolometric QLF to observational data, we also require a model for the intrinsic SED and a pre- scription for the relative obscuration of quasars at different wavelengths.

The analyses of samples of optically selected (type-I) quasars suggest that there is a luminosity dependent correlation between the X-ray and optical luminosity, which may be described in terms of the power-law index connecting the optical and X-ray flux, $\alpha_{O X} . \alpha_{O X}$ may be written (e.g. Steffen et al. 2006)

$$
\begin{aligned}
\alpha_{O X} & =-\frac{\log _{10}\left(L_{\nu}(2500 \AA) / L_{\nu}(2 \mathrm{keV})\right)}{\log _{10}(\nu(2500 \AA) / \nu(2 \mathrm{keV}))}, \\
& =-A \log _{10}\left(\frac{L_{\nu}(2500 \AA)}{\mathrm{ergs} / \mathrm{s} / \mathrm{Hz}}\right)+B,
\end{aligned}
$$

where $A, B>0$ corresponding to an anti-correlation of $\alpha_{O X}$ with optical flux. Steffen et al. (2006) estimate $A=$ $0.137 \pm 0.008$ and $B=2.638 \pm 0.24$ for an optically selected sample of quasars with no apparent intrinsic absorption or radio activity. Steffen et al. (2006) find no strong evidence for evolution of this relationship with redshift, however the data is consistent with $\alpha_{O X}$ decreasing slowly with redshift as $\sim 0.01 z$.

We adopt a modified version of the model SED presented in Hopkins, Richards, \& Hernquist (2007) to calculate the (luminosity dependent) SED for a given observed frequency, $\nu_{\mathrm{obs}}$, quasar redshift, $z$, and bolometric luminosity, $L_{\mathrm{bol}}$. This model assumes the SED to be essentially a broken power-law which mimics reprocessed emission in the NIR, the UV bump and the hard X-ray excess features with an exponential cut-off at $500 \mathrm{keV}$. The SED is normalised so that the observed relation between optical and X-ray emission described by equation (8) with $A=0.109$ and $B=1.739$ is preserved.

Note that Hopkins et al. (2007) found that the luminosity dependence of the relation between optical and X-ray emission is more important for reconciling the optical and X-ray QLFs at $0<z<5$ than the detailed modelling of the features in the SED. The anti-correlation of $\alpha_{O X}$ with $L_{\nu}(2500 \AA)$ results in a narrower spread of X-ray luminosities compared to the corresponding optical range. The model $\mathrm{X}$-ray QLF is thus steeper than the equivalent bolometric QLF.

We find that the bright end of our model X-ray luminosity function becomes too steep compared to the measurements in both the soft and hard X-ray bands when we use the Hopkins et al. (2007) SED. Including a dispersion in the bolometric corrections, which Hopkins et al. (2007) note has the largest effect on the bright end of the X-ray QLF, would smooth the QLF and somewhat alleviate this. We have chosen instead to alter the luminosity dependence of the SED model to fit the data as described below.

To achieve a good match with the bright end of the observed X-ray QLF we adopt an SED for $L_{\mathrm{bol}}>10^{14} \mathrm{~L}_{\odot}$ with $A=0.104$ and $B=1.739$. We use the same power-law indices, break-points and exponential cut-off as Hopkins et al. (2007) but have not modelled the reflection component or the NIR emission. This choice of parameters increases the power in the soft and hard X-ray bands by approximately 40 per cent at $L_{\text {bol }}=10^{14} \mathrm{~L}_{\odot}$, increasing to 45 per cent at $10^{15} \mathrm{~L} \odot$. The power at UV wavelengths changes minimally. To ensure a smooth QLF we take an average of the Hopkins et al. (2007) and the above modification for bolo- 

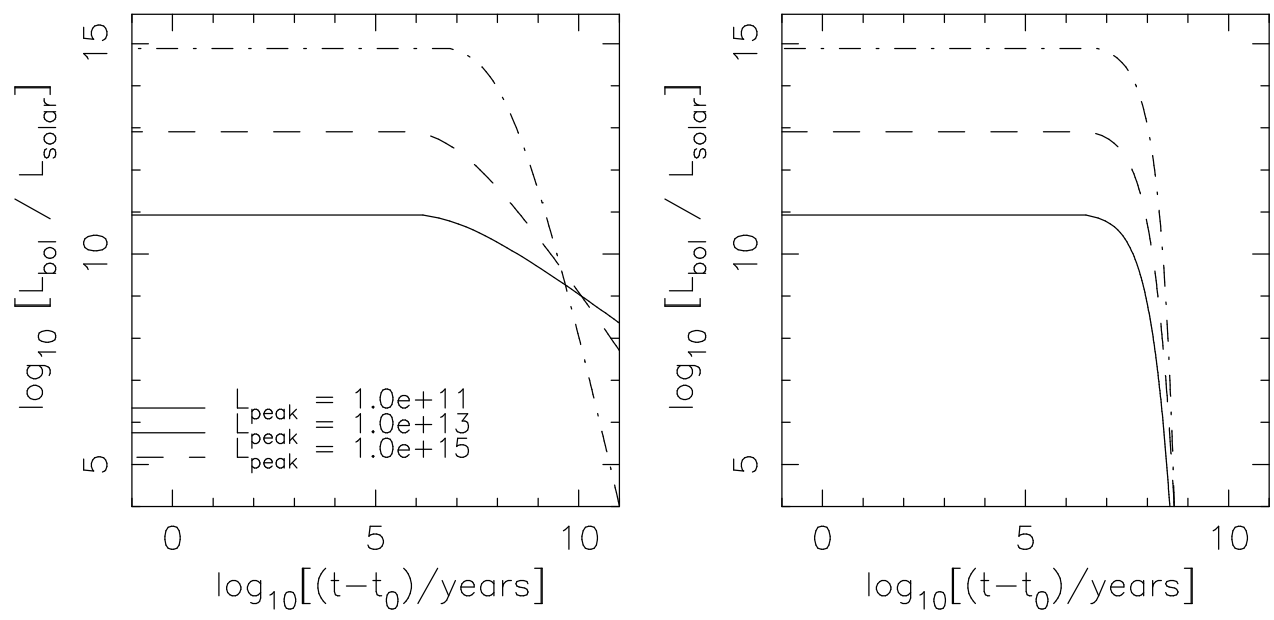

Figure 1. Light curves used to generate model QLFs for quasars with intrinsic, peak, bolometric luminosities of $10^{11}$ (solid line), $10^{13}$ (dashed line) and $10^{15}$ (dot-dashed line) $L_{\odot}$. The left panel shows the slow fading law, corresponding to equation (5) with the average lifetime $\left(t_{9}\right)$ increased by a factor of two. The right panel is for the rapid fading law $\frac{d t}{\operatorname{dlog} L}=1.7 \times 10^{7}$ yrs $\left(\frac{L}{L_{\text {peak }}}\right)^{-0.01}$ corresponding to rapid (almost exponential) luminosity independent fading.

metric luminosities between $10^{12}$ and $10^{14} \mathrm{~L} \odot$ weighted by the logarithmic bolometric luminosity.

\subsection{The effect of absorption on the SED}

Light emitted from the accretion disk of the AGN is reprocessed during transmission through its host galaxy, the IGM and our own galaxy. At the X-ray energies and redshifts we are mainly interested in (observed frame $0.5-10 \mathrm{keV}$ ) the absorption of X-rays by neutral hydrogen in the IGM can be neglected. Galactic absorption is generally corrected for, and therefore we are mainly concerned with absorption at the quasar redshift. Assuming that emission at all wavelengths is obscured by the same body of gas, the absorption at a particular wavelength can be determined given a column density distribution of neutral hydrogen (which may depend on parameters of the system) a dust-to-gas ratio and a reddening law for the dust absorption. We again adopt similar assumptions as Hopkins et al. (2005b) for our model, which we discuss in the following two sections.

\subsubsection{Column density distribution of the absorbing gas}

The column density distribution of absorbing gas is expected to be related to the fading law; gas both feeds the $\mathrm{BH}$ and obscures the radiation released. However the astrophysical relationship is complicated and depends on, for example, the surrounding star formation and the geometry of the accretion disc. Hopkins et al. (2005a) have used the numerical simulations of quasars fuelled by gas accretion by Di Matteo et al. (2005) to construct a parametrised luminosity and time dependent model for the obscuring column density. The probability $P$ that a quasar is obscured by a given total hydrogen column density $N_{\mathrm{H}}$ depends on the amount of time a quasar remains in a given accretion phase. The simulated data is fit with a log-normal distribution

$$
\begin{aligned}
P\left(N_{\mathrm{H}}, L\right) & =\frac{1}{\sigma_{\mathrm{N}_{\mathrm{H}}} \sqrt{2 \pi}} \exp \left\{-\left[\log _{10}\left(N_{\mathrm{H}} / \bar{N}_{\mathrm{H}}\right)\right]^{2} /\left(2 \sigma_{N_{\mathrm{H}}}^{2}\right)\right\} \\
\bar{N}_{\mathrm{H}} & =10^{21.9} \mathrm{~cm}^{-2}\left(\frac{L}{10^{11} \mathrm{~L}_{\odot}}\right)^{0.43} \\
\sigma_{N_{\mathrm{H}}} & \approx 1.2 .
\end{aligned}
$$

Note that the mean column density, $\bar{N}_{\mathrm{H}}$, and dispersion, $\sigma_{\mathrm{N}_{\mathrm{H}}}$, are predominantly determined by the instantaneous bolometric luminosity of the quasar $L$.

Hopkins et al. (2005a) also demonstrated that the shape of this distribution for quasars with a B-band luminosity above $10^{11} \mathrm{~L}_{\odot}$ is consistent with a statistical analysis of the reddening found toward SDSS quasars in Hopkins et al. (2004). This analytic fit is derived from simulations of gaseous disc galaxies, and of course may not be representative of the typical accretion rate onto black holes at all redshifts. However at the redshifts that we are applying it $(z>2)$ galaxies are expected to be gas rich.

\subsubsection{Dust and gas absorption}

Since we are predominantly comparing our model to optical data quoted in UV magnitudes we have used the reddening law presented in Gaskell et al. (2004) to model dust and gas absorption. The reddening law is normalised such that the optical depth in the V-band (5500 $\AA$ ) is the same as for an SMC-like reddening curve [taken from (Pei 1992)] for a galaxy with the metallicity of the Milky Way. This results in a V-band optical depth identical to that adopted by Hopkins et al. (2005b) who assumed a SMC-like reddening curve with a gas-to-dust ratio as for the Milky Way. At X-ray wavelengths the absorption is dominated by photo-electric absorption and Compton scattering by hydrogen. Following Hopkins et al. (2005b) we assume an average neutral gas 
fraction of 0.35 and assume that the ionised component contributes to the photoelectric absorption, but not the optical reddening or Compton scattering. We note that this model makes the simplistic assumption that the distribution of gas and dust is spatially uniform.

\subsection{The model luminosity function taking absorption into account}

We now turn to calculating the observed luminosity function for a given redshift $z$ and observed wavelength $\nu$ (or more usefully for comparison with X-ray observations, wave-band $\left.\nu_{a} \rightarrow \nu_{b}\right)$.

Given the probability that a quasar with a given intrinsic bolometric luminosity $L^{\prime}$ is obscured by a given column density, the probability that the intrinsic specific luminosity $L_{\nu}^{\prime}$ is observed to have a specific luminosity $L_{\nu}$, $P\left(L_{\nu} \mid L_{\nu}^{\prime}\right) d \log L_{\nu}^{\prime}$, may be written

$$
\begin{aligned}
& P\left(L_{\nu} \mid L_{\nu}^{\prime}\right) d \log L_{\nu}^{\prime} \\
= & P\left(N_{\mathrm{H}}=\frac{1}{\sigma_{\nu}} \log \left(\frac{L_{\nu}^{\prime}}{L_{\nu}}\right), L^{\prime}\right) \frac{d \log _{10} N_{\mathrm{H}}}{d \log L_{\nu}^{\prime}} d \log L_{\nu}^{\prime}, \\
= & P\left(N_{\mathrm{H}}=\frac{1}{\sigma_{\nu}} \log \left(\frac{L_{\nu}^{\prime}}{L_{\nu}}\right), L^{\prime}\right) \frac{\log _{10}(e)}{N_{\mathrm{H}} \sigma_{\nu}} d \log L_{\nu}^{\prime},
\end{aligned}
$$

where $\sigma_{\nu}$ is the absorption cross section at $\nu$ in units $\mathrm{cm}^{2}$. The absorbed luminosity function at an observed wavelength $\nu$ and redshift $z,\left.\frac{d n}{\operatorname{dog} L_{\nu}}\right|_{o b s}(z)$, can be calculated by integrating over the unabsorbed bolometric QLF, $\frac{d n}{\operatorname{dog} L_{\nu}}$,

$$
\begin{aligned}
\left.\frac{d n}{d \log L_{\nu}}\right|_{o b s}(z)= & \int_{\log \left(L_{\nu(1+z)}^{\prime}\right)=\log \left(L_{\nu(1+z)}\right)}^{\infty} \frac{d n}{\operatorname{dlog} L_{\nu(1+z)}^{\prime}}(z) \\
& P\left(L_{\nu(1+z)} \mid L_{\nu(1+z)}^{\prime}\right) d \log L_{\nu(1+z)}^{\prime} .
\end{aligned}
$$

The convolution is identical if we want to consider the luminosity function in band $\left(\nu_{a}, \nu_{b}\right)$, except the change of variable in equation (10) becomes $\frac{d \log _{10} N_{H}}{d \log L_{\text {band }}^{\prime}}=\frac{1}{N_{H}<\sigma_{\nu}>}$. Here $\left\langle\sigma_{\nu}\right\rangle$ is the average absorption cross section in the band, weighted by the observed luminosity $\nu L_{\nu} e^{-\sigma_{\nu} N_{H}}$,

$$
<\sigma_{\nu}>=\frac{\int_{\nu_{a}}^{\nu_{b}} d \nu \sigma_{\nu} \nu L_{\nu} e^{-\sigma_{\nu} N_{H}}}{\int_{\nu_{a}}^{\nu_{b}} d \nu \nu L_{\nu} e^{-\sigma_{\nu} N_{H}}}
$$

\subsection{The blowout phase}

The probability distribution of the absorbing hydrogen column density given in equation (9) will not be an adequate description when the luminosity is close to the peak luminosity, as much of the gas is expected to be blown away by radiation pressure. As discussed by Hopkins et al. (2005a) the true column density distribution in the simulations is bimodal, with quasars in the last e-folding of growth having a much lower obscuring column density distribution. This so called "blow-out" phase lasts approximately 10 per cent of the total time the quasar spends accreting. Hopkins et al. (2005a) suggest that this bi-modality can explain why optically selected quasars are observed to have lower obscuring column densities for brighter quasars (e.g. Ueda et al. 2003), counter to the positive correlation between $L$ and $\bar{N}_{H}$ in equation (9).

We have taken a simple approach to modelling such a blowout phase; we assume that a fraction $f_{\text {blowout }}=0.1$ of quasars within a factor of $e$ of their peak luminosity experience no intrinsic absorption. The resulting absorbed luminosity function is then larger than that in equation (10) by a term $\frac{d n}{d \log L_{\nu}}\left(\frac{L}{L_{\text {peak }}}>\frac{1}{e}\right) \times f_{\text {blowout }}$, where $\frac{d n}{d \log L_{\nu}}\left(\frac{L}{L_{\text {peak }}>} \frac{1}{e}\right)$ is the QLF of sources with specific luminosity $L_{\nu}$ that have total bolometric luminosity within $e$ of their peak luminosity. $\frac{d n}{\operatorname{dlog} L_{\nu}}\left(\frac{L}{L_{\text {peak }}>\frac{1}{e}}\right)$, increases with $L_{\text {bol }}$, and approaches the original (without blow out phase) value of $\frac{d n}{\operatorname{dog} L_{\nu}}$ for large $L_{\text {bol }}$. This prescription for the blowout phase essentially puts a lower limit on the ratio of the absorbed to unabsorbed luminosity function at high luminosities equal to $f_{\text {blowout }}$.

The parameters of the blow-out phase affect the relative number of bright optical and X-ray luminous sources. In particular, if we didn't include a blow-out phase (and were therefore assuming that more of the bright optical sources are obscured) then we would predict more X-ray bright sources. However we find that the absorption in the soft X-ray band behaves similarly to that in the optical and therefore if we did not include the blow-out phase it would be very difficult to reproduce the space density of quasars that are bright in soft X-rays.

\section{EVOLUTION OF THE MODEL LUMINOSITY FUNCTION IN DIFFERENT WAVEBANDS AND COMPARISON WITH OBSERVATIONAL DATA}

\subsection{Calibrating the merger-driven model for the evolution of the luminosity function at intermediate redshift with observations}

We vary the parameters $\left(t_{q}, \epsilon_{o}, L_{\text {peak,min }}\right)$ in order to obtain an acceptable fit to the observed luminosity functions at rest-frame $1450 \AA$, and in the soft and hard X-ray bands. We determine the normalisation of our models (governed by $t_{q}$ and $\epsilon_{o}$ ) by comparing to the observed optical QLF, as these constraints span the broadest redshift range. However, as we will discuss below, the optical data has little power to constrain $L_{\text {peak,min }}$ and constraints on this value come from low-luminosity X-ray observations alone.

We find that assuming the luminosity independent fading law in equation (7), it is difficult to accommodate values of $\alpha_{L}$ much less than zero, due to the intrinsic steepness of the cosmological merger rate we are adopting for the quasar formation rate. However for $\alpha_{L}=-0.01, t_{q}=$ $1.74 \times 10^{7}$ years and $\epsilon_{0}=10^{-5.05}$ we recover a good fit to the optical QLF at $z=2-6$ (see Figure 2). This value of $\alpha_{L}$ corresponds to a light-curve for which the luminosity drops off almost exponentially with time, we will therefore refer to this model as the "rapid fading" model. The corresponding light curve is compared to the Hopkins et al. (2005a) light curve (with some adjustment described below) in Figure 1. The Hopkins et al. (2005a) fading law, with appropriate $\epsilon_{o}$, also provides a reasonable fit to the optical data at $z=2-6$ as described by equation (5). To put it on equal footing with what we have done for the fit with the rapid fading law we allow the characteristic time, $t_{9}$, to vary. We find that 

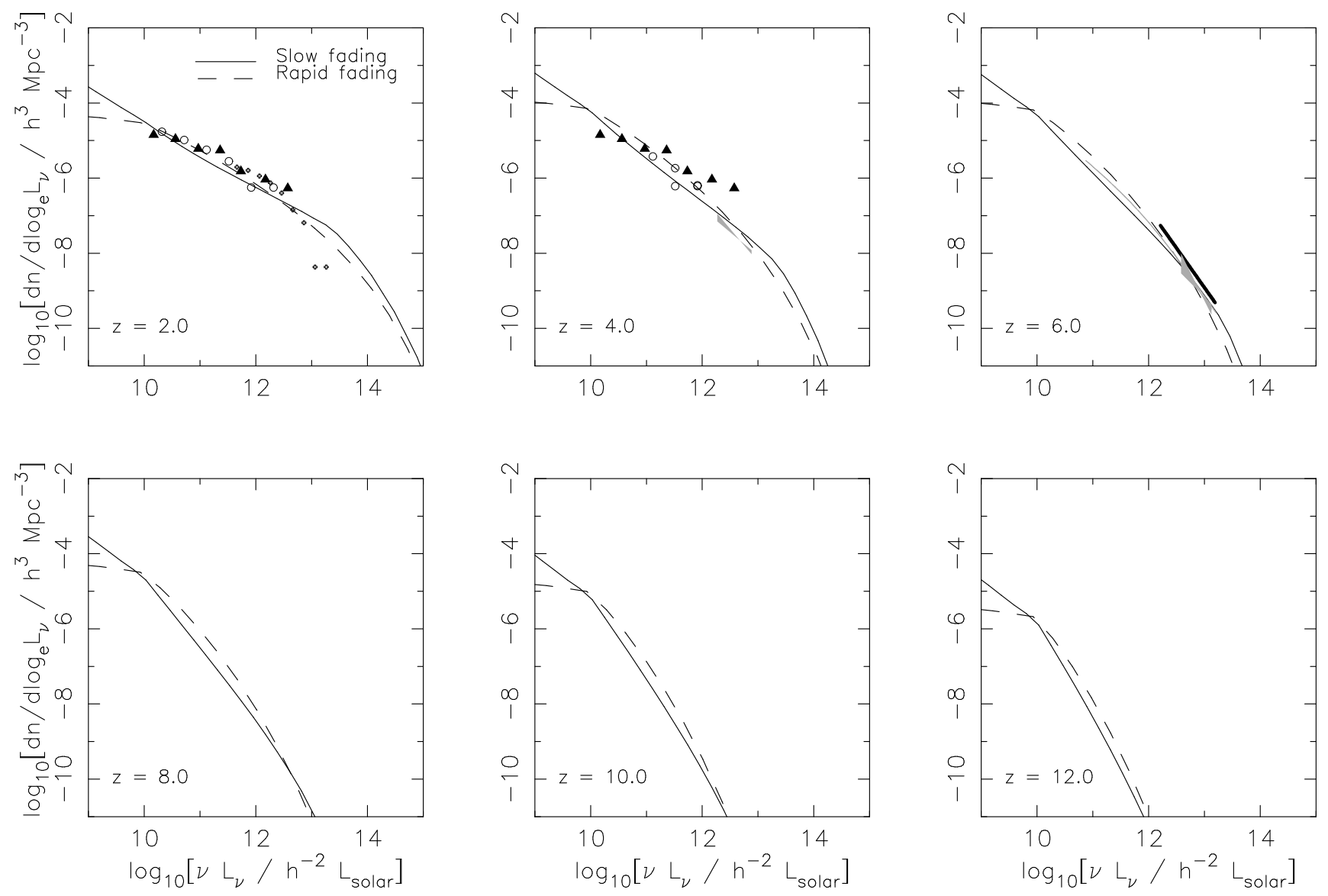

Figure 2. Model rest-frame $1450 \AA \mathrm{QLF}$ at $z=2,4,6,8,10,12$ for a merger driven model with a slow fading law (solid line) and the rapid fading law (dashed line) as described in the text. Note that we have neglected absorption by neutral gas in the IGM which will affect what is observed in practice at $z \gtrsim 6$. The hollow circles are the measurement of the faint end QLF from the COMBO-17 survey (Wolf et al. 2003). The black triangles at $z=2,4$ show the luminosity function measured by Bongiorno et al. (2007) in the redshift interval $2.1<z<3.6$. The small black diamonds at $z=2$ are the results from Croom et al. (2004) using the SED from Hopkins et al. (2007) to convert from B-band to rest frame $1450 \AA$ luminosities. The grey bow-ties represent the constraints on the bright end QLF measured in the SDSS survey (Fan et al. 2002, 2004). The thick black line at $z=6$ is the fit from Jiang et al. (2007). The thin grey line at $z=6$ is the Shankur \& Mathur (2006) constraint on the slope of the QLF from the dearth of X-ray sources.

the fit is improved when $t_{9}$ is increased by a factor of 2 to $2 \times 10^{9}$ years. A slightly larger value of $\epsilon_{o}=10^{-4.97}$ than for the rapid fading model is required to offset the smaller amount of time that bright quasars spend at their Eddington luminosity. We refer to the model with this fading law as the "slow fading" model. Since the two models provide comparable fits to the optical data, the optical data alone appears to offer little power to constrain the luminosity dependence of the fading law.

Note that there is an excess in the predicted number of optically bright quasars at $z=2$ for each fading law. The most plausible explanation for this discrepancy between our model and the data is probably our neglect of AGN feedback. AGN inject large amounts of heat into their surroundings (e.g. Dunn \& Fabian 2006; Best et al. 2007) and should be capable of suppressing cooling flows in dark matter halos with masses above $\sim$ few $\times 10^{13} h^{-1} \mathrm{M}_{\odot}$ - resulting in the formation of groups and clusters of galaxies rather than super-sized quasars in very large dark matter halos (see, e.g. Sijacki \& Springel 2006; Sijacki et al. 2007; Rines et al. 2007). This effect is expected to be more prominent at low redshifts when the typical masses of merging dark matter halos is largest. Indeed most semi-analytic models of the quasar population require some arbitrary high mass cut-off in order to fit to the low redshift data (e.g. Kauffmann \& Haehnelt 2000; Cattaneo et al. 2007). To capture this effect in our model in a redshift dependent way would be difficult and we do not attempt this here. It is also for this reason that our model, and similar semi-analytic models for the QLF, do not reproduce quasar luminosity function below $z \sim 2$ very well.

With $t_{q}$ and $\epsilon_{o}$ chosen to reproduce the optical luminosity functions we find fairly good agreement with the observed $z \sim 2 \mathrm{QLF}$ in the hard X-ray band, and consistency with the constraints on the $z \sim 4$ hard X-ray QLF (see Figure 3). The value of the minimum $\mathrm{BH}$ mass (or minimum peak luminosity) does not affect the QLF at the luminosities relevant for optical constraints, however the constraints on the X-ray QLF are approximately 2 orders of magnitude deeper at $z=2$ than in the optical. We find that a cut-off at $L_{\text {peak }}=10^{11} L_{\odot}$ is required for the rapid fading model in order to avoid over-predicting the number of sources in 

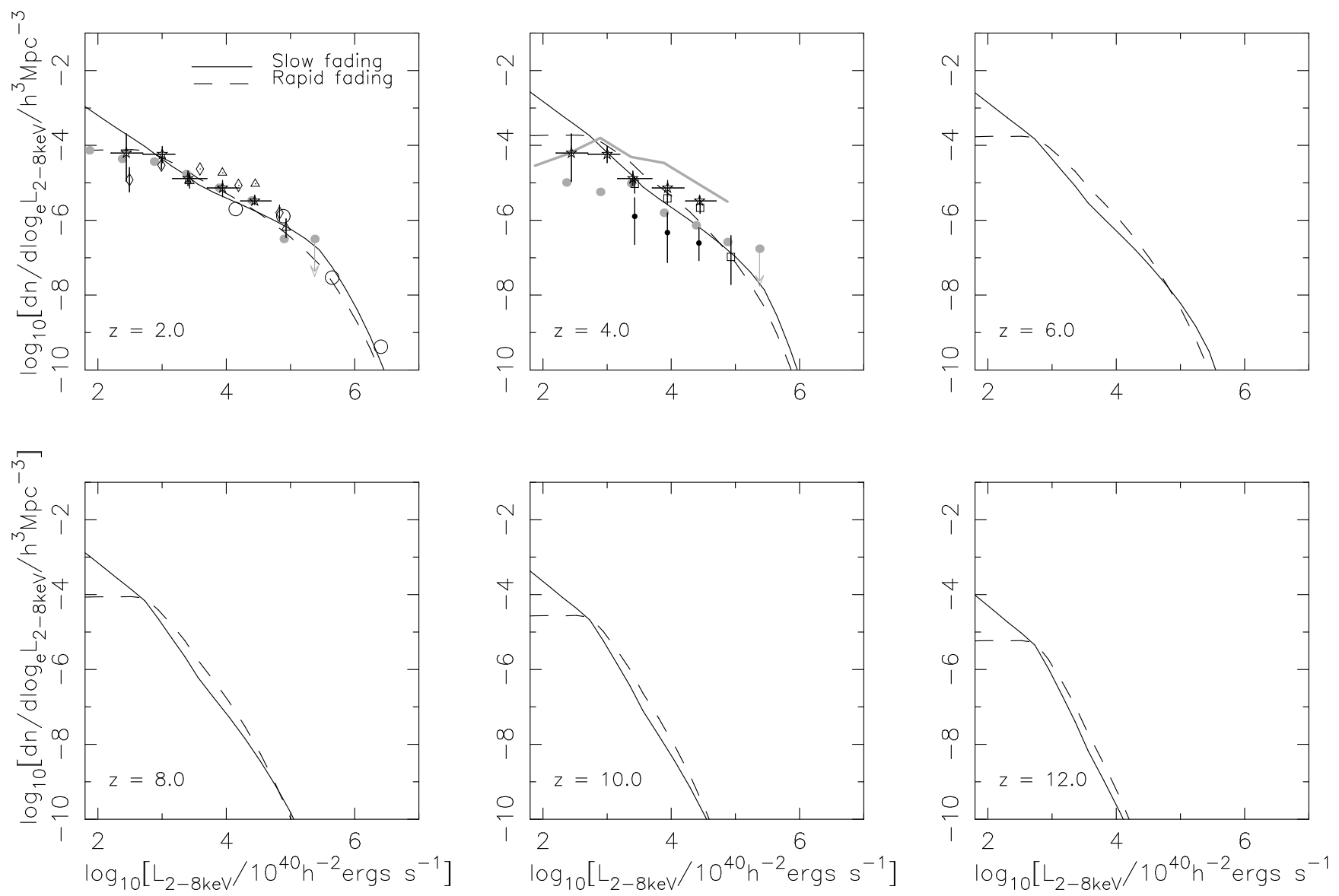

Figure 3. Model rest-frame $2-8 \mathrm{keV}$ QLF at $z=2,4,6,8,10,12$ for a merger driven model with slow fading (solid line) and rapid fading (dashed line) as in Figure 2. The filled grey circles are estimates from Barger et al. (2005) and the hollow grey circles the rest frame $2-10 \mathrm{keV}$ data from Ueda et al. (2003). The thick grey line in the $z=4$ panel corresponds the estimate from Barger et al. (2005) assuming that all spectroscopically unconfirmed sources lie in the $z=3-5$ bin. The black markers are the hard X-ray QLF from Silverman et al. (2007); the hollow diamonds for $1.5<z<2$, the hollow triangles for $2<z<3$, the hollow squares for $3<z<4$ and the filled circles for $4<z<5.5$. The stars with horizontal and vertical error bars are the measurement for $2.5<z<3.5$ from Aird et al. (2008).

the lowest luminosity bin in the hard X-ray QLF at $z=2$. We apply the same cut-off in $L_{\text {peak }}$ to the Hopkins-fading model and find it over-predicts the rather shallow faint end of the observed luminosity function at $z=2$ in the hard Xray band (see Figure 3). Note that an Eddington luminosity of $10^{11} \mathrm{~L}_{\odot}$ corresponds to a $\mathrm{BH}$ mass $\sim 3.10^{6} \mathrm{M}_{\odot}$ and there is certainly evidence for the existence of BHs with masses smaller than this (e.g. Greene \& Ho 2007a).

In Figure 4 we plot our model observed frame $0.5-2 \mathrm{keV}$ QLF and compare it to the results of Mivaii et al. (2000), Miyaji et al. (2001) and Hasinger et al. (2005). Even with the increased power at X-ray wavelengths for the brightest sources, we still seem to under-predict the space density in the brightest bins at $z=2$ and 4 . Note, however that the highest $\mathrm{L}$ data point is at each redshift is calculated from only once source with an extremely high soft X-ray luminosity of $\sim 10^{14} \mathrm{~L}_{\odot}$, and therefore this discrepancy is probably not significant.

We find that whilst the normalisation determined from the optical constraints reproduces the counts well at intermediate luminosities, our models consistently overpredict the number of faint sources at $z=4$, and to a lesser extent at $z=2$, when compared to the type-1 AGN luminosity function of Hasinger et al. (2005). This inconsistency may be partially attributable to the restriction of the Hasinger et al. (2005) to type-1 (unobscured) AGN and incompleteness of the optical identification. If we take the data at face value, then given that the merger-rate of galaxies steepens significantly toward higher redshifts, then the Hasinger et al. (2005) data suggests that for a merger-driven model of quasar activity $L_{\text {peak,min }}$ must be increased by approximately an order of magnitude to $10^{12} \mathrm{~L}_{\odot}$. Such a cut-off brings the rapid fading model into agreement with the hard and soft X-ray data at $z=2,4$, and eases, though does not eradicate, the excess of faint objects predicted by the slow fading model. This modification would not alter the agreement of either model with the optical constraints.

Invoking a cut-off at $L_{\text {peak,min }}=10^{12} \mathrm{~L}_{\odot}$ corresponds to a minimum mass of active black holes of $\sim 3 \times 10^{7} \mathrm{M}_{\odot}$ in our model. Whilst the mass function of local BHs may turn over at around this value (e.g. Greene \& Ho 2007a b), this trend is highly uncertain and direct measurements of $\mathrm{BH}$ masses at higher redshift are not yet available.

However, it appears possible that the current surveys 

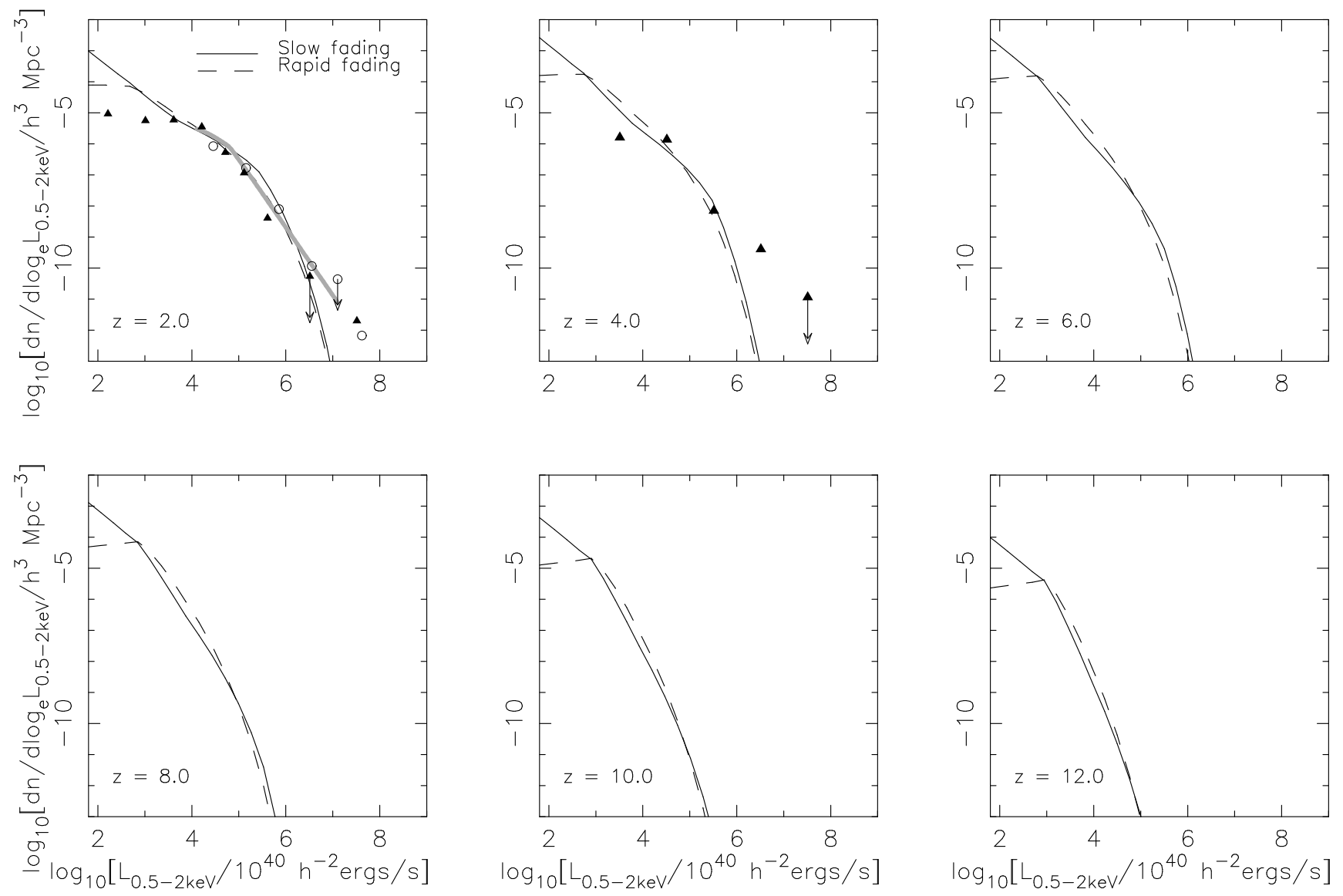

Figure 4. Observed frame $0.5-2 \mathrm{keV}$ QLF at $z=2,4,6,8,10,12$ for the same models as in Figure 3 . The thick grey line is the best-fit luminosity dependent density evolution model from (Miyaji et al. 2000) (LDDE2). The hollow markers are the binned luminosity function for $z=1.6-2.3$ from Mivaii et al. (2001). The black triangles denote the type-I AGN QLF from (Hasinger et al. 2005).

have underestimated the space density of faint sources at $z=4$ (Aird et al. 2008). Note that if the measurements of a small space density of faint hard and soft X-ray selected quasars at $z=4$ and the sudden drop in the density of X-ray quasars between $z=2$ and 4 consolidate, then the density of high redshift X-ray quasars, and therefore the number of sources detectable by the next generation of $\mathrm{X}$ ray satellites, may be lower than we are predicting with our fiducial merger-driven model. In the discussion that follows we will also discuss results for a slow and rapid fading models with $L_{\text {peak } \min }=10^{12} \mathrm{~L}_{\odot}$.

\subsection{Extrapolating the evolution of the luminosity function in the merger-driven model to very high redshift}

A prominent feature in our models of the high redshift hard and soft X-ray QLFs is the break at low luminosities in the rapid fading model due to the cut-off $L_{\text {peak,min }}=10^{11} \mathrm{~L}_{\odot}$. No such break is observed for the slow fading model where the sources with $L_{\text {peak }}=L_{\text {peak,min }}$ fade gradually. Above the break, the X-ray QLF exhibits little dependence on the fading law. This is because at high redshift the dependence of the merger rate on luminosity in our model results in a luminosity function with steeper slope than the fading rate of sources for either fading model. There exists therefore a degeneracy in the prediction of the number of faint sources between the slope of the assumed fading law and the minimum peak luminosity. Values of $L_{\text {peak,min }}<10^{11} \mathrm{~L}_{\odot}$ are certainly plausible at high redshift; theoretical models for the formation of the first BHs suggest that seed black holes with masses between 100 and $10^{5} \mathrm{M}_{\odot}$ may form as early as $z=20$ (e.g. Madau \& Rees 2001; Volonteri 2006). In Section 6 we explore the possibility of a lower value of $L_{\text {peak,min }}=10^{9} \mathrm{~L}_{\odot}$ $\left(M_{\mathrm{bh}, \min } \approx 3 \times 10^{4} \mathrm{M}_{\odot}\right)$.

\subsection{Alternate models for the early growth of supermassive black holes}

The mechanism for the growth of very high redshift BHs is very uncertain. Estimates for the formation of the first, or seed, BHs vary widely. BHs with masses $10^{2}-10^{3} \mathrm{M}_{\odot}$ may form from population-III remnants at $z=20$ or earlier (Madau \& Rees 2001). To grow BHs as large as a few $\times 10^{9} \mathrm{M}_{\odot}$ by $z \sim 6$ from a pop-III remnant mass seed requires more or less continuous accretion if the accretion rate Eddington limited (e.g. Archibald et al. 2002). Li et al. (2007b) find that in a merger-driven scenario, BHs large enough to power the brightest $z \sim 6$ quasars may be grown from seed BHs with mass $10^{5} \mathrm{M}_{\odot}$. In this model most progenitors in the (hierarchical) merging history of the $\mathrm{SMBH}$ grow at sub-Eddington rates for the majority of the time. 
The overall growth, however, is dominated by the fastest growing BHs at each step in the hierarchy. In aggregate the growth is similar to almost continuous Eddington limited accretion of a single seed BH. Following Rhook \& Haehnelt (2006) we consider a passive evolution scenario in which a fixed comoving density of BHs evolve by accreting at their Eddington limit with a fixed duty cycle $f_{\text {duty }}$. In this model for the early $\mathrm{BH}$ growth, the ensemble average luminosity of a BH increases with decreasing redshift as,

$$
\begin{aligned}
L & =L(z=6) e^{-\left(t_{z=6}-t_{z}\right) f_{\mathrm{duty}} / \kappa} \\
\kappa & =\frac{c \sigma_{e}}{4 \pi G m_{\mathrm{p}}} \frac{\epsilon_{\mathrm{acc}}}{1-\epsilon_{\mathrm{acc}}} \\
& \simeq 5 \times 10^{8} \mathrm{yrs} \frac{\epsilon_{\mathrm{acc}}}{1-\epsilon_{\mathrm{acc}}}
\end{aligned}
$$

where $\sigma_{e}$ is the Thompson scattering cross section.

The exponential growth of BHs in this model makes it unsuitable for modelling the QLF below $z \sim 6$. We therefore consider this model as a possible alternative to the mergerdriven evolution only at $z>6$. We adopt the rapid fading merger-driven model with $L_{\text {peak,min }}=10^{9} \mathrm{~L}_{\odot}$ at $z<6$.

Extending the absorption laws used in the previous section, which were determined from estimates of the average column density along the line of sight to a pair of merging galaxies, is probably not meaningful for continuously accreting BHs. We therefore simply assume that a fixed, luminosity independent, fraction of the accreting BHs are unobscured, and that the rest are Compton-thick (completely obscured in the optical and X-ray). The total duty fraction is then made up of the duty fraction of BHs that are accreting in an X-ray luminous phase $f_{\text {lum }}$ and those that are accreting in a Compton thick phase $f_{\text {obsc }}$.

Note that for a model in which the BHs are growing continuously (although with some duty cycle) the concept of a fading law is also no longer meaningful since we are assuming that there is always enough gas for the $\mathrm{BHs}$ to accrete at their Eddington limit.

We have explored the evolution above $z=6$ for two values of the total duty fraction, $f_{\text {duty }}=0.1,1.0$. The fraction of obscured sources at high redshift is unknown but is certainly significant at lower redshift (e.g. Risaliti et al. 1999; Martínez-Sansigre et al. 2007). We assume that half of the sources are totally obscured in each case, which is consistent with the constraints from population synthesis models of the CXRB (Gilli et al. 2007).

For our models with Eddington limited $\mathrm{BH}$ growth at $z>6$ our assumptions governing the accretion rate and intrinsic absorption change discontinuously at $z=6$ in such a way that the observed hard X-ray QLF changes smoothly. This alternate model for the early growth of SMBHs should thus be regarded only as a demonstration of the uncertainty in the growth rate of $\mathrm{BHs}$, and therefore the number of detectable X-ray quasars, at very high redshifts.

In Section 5.3 we explore the predictions for the number of quasars detectable at X-ray wavelengths for both passive and merger-driven $\mathrm{BH}$ growth scenarios. We first compare our merger-driven models to the constraints from the observed flux distribution of X-ray sources and the CXRB.

\section{X-RAY NUMBER COUNTS AND REDSHIFT DISTRIBUTION AT FAINT FLUX LEVELS AND THE EARLY GROWTH OF SUPERMASSIVE BLACK HOLES}

\subsection{X-ray source counts and redshift distributions}

The main aim of our modelling is to predict the X-ray source counts and the corresponding redshift distribution at faint flux levels.

The X-ray source counts ("the $\log N-\log S$ relationship") are well described by a double power-law, with the slope flattening for fluxes below $\sim 1.5 \times 10^{14} \mathrm{erg} \mathrm{s}^{-1}$ in the soft X-ray band and below $\sim 4.5 \times 10^{15} \mathrm{erg} \mathrm{s}^{-1}$ in the hard X-ray band (e.g. Cowie et al. 2002; Moretti et al. 2003). More recently, the constraints on the number counts have been extended to lower sensitivities by fluctuation analyses of the unresolved background. These constraints generally still allow for an upturn in the $\log N-\log S$ relationship below the detection limit for resolved sources (e.g. Hickox \& Markevitch 2006).

In the top panel of Figure 5 we plot the source counts as a function of limiting flux sensitivity in the hard X-ray $(2-10 \mathrm{keV})$ band and in Figure 6 for the soft $(0.5-2 \mathrm{keV})$ band. The left panels show the results for the slow fading model, the middle for the rapid fading model with $L_{\text {peak,min }}=10^{11} \mathrm{~L} \odot$ and the right for the rapid fading with $L_{\text {peak,min }}=10^{9} \mathrm{~L} \odot$. In each case the over-plotted data is taken from the fluctuation analysis of Mivaii \& Griffiths (2002) for the Chandra Deep Field North (CDF-N). Recall that below $z=2$ we have supplemented our model for the X-ray QLF with a fit to the observed QLFs (calculated at discrete redshifts $z=0.01,0.6,1.2)$. We have used the PLE model from Barger et al. (2005) for the hard X-ray band and the LDDE2 model from (Mivaii et al. 2001) for the soft $\mathrm{X}$-ray band. Therefore by construction our models should reproduce the observed source counts if our model is a reasonable approximation at $z \geqslant 2$.

Our models are consistent with the constraints on the hard X-ray counts for both the rapid and slow fading laws. However, for the soft X-ray counts only the rapid fading models are consistent with the observed counts. Using the slow fading law our model slightly overshoots the faint soft X-ray counts and lies above the limit suggest by the fluctuation analysis of Mivaji \& Griffiths (2002), as is expected from the inconsistency with the measured QLF at $z \sim 2$ discussed in Section 4.1. For the slow and rapid fading models with $L_{\text {peak,min }}=10^{12} \mathrm{~L}_{\odot}$, the number counts below the current detection levels are reduced, bringing the slow fading model into agreement with the fluctuation analysis and resulting in a flat source distribution at low flux levels for the rapid fading model.

In the lower panel of Figure 5 we plot the normalised redshift distribution for the source counts above three flux/sensitivity levels. The grey line is for the approximate sensitivity of current X-ray satellites $\left(3 \times 10^{-16} \mathrm{ergs} / \mathrm{s} / \mathrm{cm}^{2}\right)$, the solid black line for an order of magnitude fainter $(3 \times$ $\left.10^{-17} \mathrm{ergs} / \mathrm{s} / \mathrm{cm}^{2}\right)$ and the dotted line for the goal sensitivity of a 1 Ms observation with XEUS $\left(3 \times 10^{-18} \mathrm{ergs} / \mathrm{s} / \mathrm{cm}^{2}\right.$, but see the discussion in Section 5.3). We note the strong dependence of the redshift distribution on the fading law. For the slow fading model the probability for source detection peaks at $z \sim 1-3$ for each of the chosen flux limits, with 

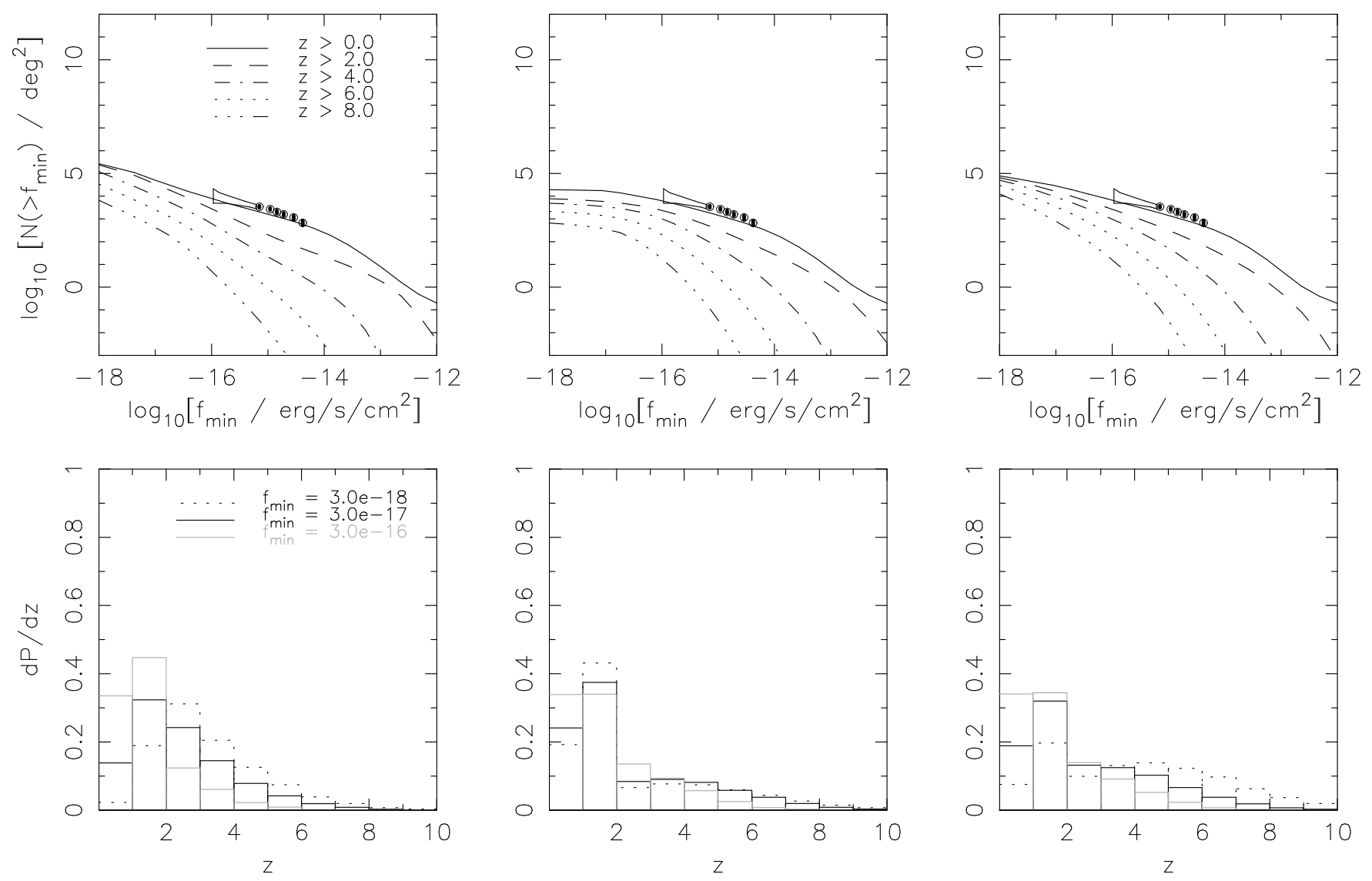

Figure 5. Results for the expected number counts of sources in the $2-10 \mathrm{keV}$ band for the model with slow fading (left) and rapid fading with $L_{\text {peak,min }}=10^{11} \mathrm{~L}_{\odot}$ (middle) and $L_{\text {peak,min }}=10^{9} \mathrm{~L}_{\odot}$ (right). Note that the models have been supplemented with observational QLFs below $z=2$ as described in the text. The top panel shows the counts integrated from $z=0,2,4,6,8,10$ to $z=20$ as labelled. The hollow markers with error bars (bow-tie) are the estimate of the resolved (unresolved) source density in the Chandra deep field by Miyaji \& Griffiths (2002). The bottom panel shows the redshift distribution of the counts for sources above flux levels of $3 . e-18$ (dotted line), 3.e - 17 (solid line) and 3.e - 16 (grey line) in cgs units.
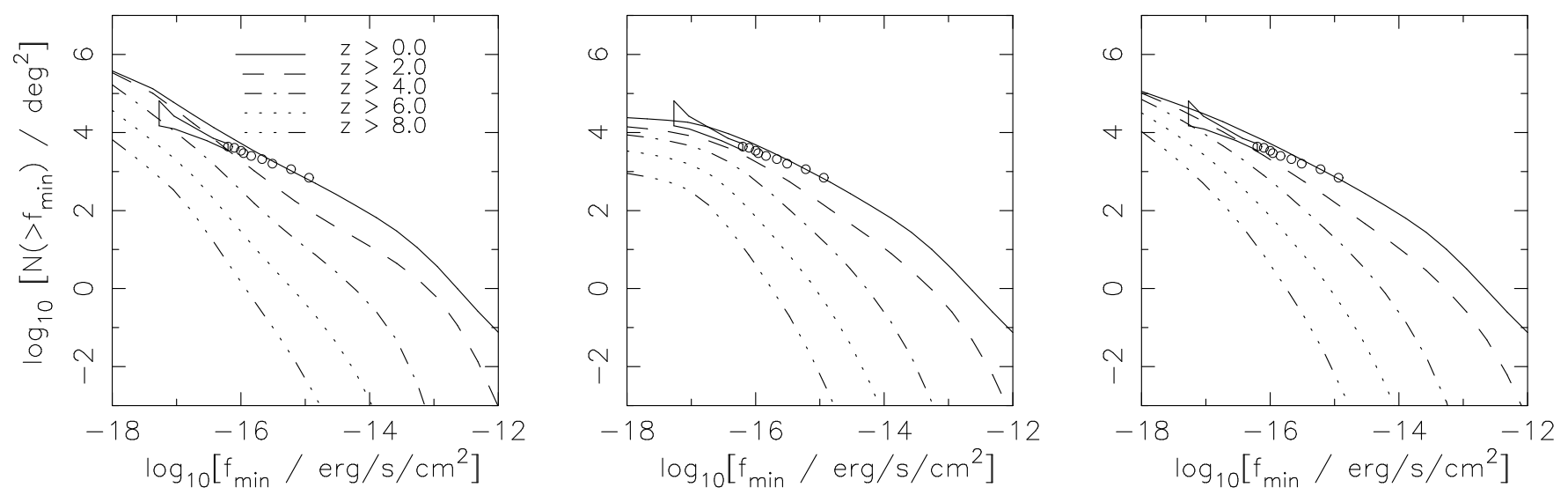

Figure 6. Results for the expected number counts in the $0.5-2 \mathrm{keV}$ band for the models in Figure 5 . At $z<2$ the models have been supplemented with the LDDE2 fit to the measured 0.5 - $2 \mathrm{keV}$ QLF from Miyaji, Hasinger \& Schmidt (2000). The hollow markers with error bars (bow-tie) are the estimate of the resolved (unresolved) source density in the Chandra deep field by Miyaji \& Griffiths (2002). 
the peak shifting to slightly higher redshift for the lower flux limits. For this model the source counts remain dominated by fading objects at intermediate redshifts where the space density of quasars is at its peak.

For the rapid fading models the source distribution is sharply peaked at $z \sim 1$ for the brightest flux limit. This is not surprising: this is where most of the known sources lie and we have used the observed data at low redshifts as our model QLF. At fainter flux limits a second broader peak around $z \sim 4$ emerges for the rapid fading models. The broad high redshift peak at faint flux level in our model is due to the steepening of the merger rate at $z>2$ combined with the increase in the comoving volume element out to $z \sim 3.5$.

The emergence of two distinct populations at faint flux levels, at low and high redshift, can be explained physically by an evolution of the Eddington ratio and the characteristic lifetime with redshift. Observationally, optically selected high redshift and/or high luminosity AGN display near Eddington accretion rates (see, e.g. McLure \& Dunlop 2004; Kollmeier et al. 2006), whereas hard X-ray selected sources at $z<1$ appear to have much lower accretion rates (e.g. Babić et al. 2007). At decreasing flux levels surveys will become sensitive to both the very faint sources accreting at low redshift and sources accreting at higher rates at high redshift, and therefore the source distribution should become double peaked.

The predicted high redshift peak for the rapid fading model is, as expected, more prominent for the scenario where more abundant, smaller mass BHs may power quasars. For the case with $L_{\text {peak,min }}=10^{9} \mathrm{~L} \odot$ almost a quarter of sources are predicted to be above $z \sim 6$ at the lowest flux level plotted. The fraction of sources expected above $z=4,6,8$ for each of the models at the three flux limits shown in Figure 5 is tabulated in Table 1.

\subsection{The integrated X-ray background}

The Cosmic X-ray Background (CXRB) and its unresolved component provides an important consistency check for models of the number of faint sources.

We may calculate the contribution of quasars in a redshift band $(z, z+d z)$ to the X-ray background in an observed band $X$ by integrating over the QLF,

$\frac{d f_{X}}{d z} d z=\left.\int_{L_{X}=f_{\min } 4 \pi D_{L}^{2}(z)} \frac{d n}{d \log L_{X}}\right|_{\mathrm{obs}}(z) \frac{d V_{c}}{d \Omega} \frac{L_{X}}{\left[D_{L}(z)\right]^{2}} d \log L_{X}$,

where $D_{L}$ is the luminosity distance and the limits of integration are determined from the limiting sensitivity $f_{\min }$.

In Figures 7 and 8 we compare the total CXRB flux predicted for the slow fading model, and the rapid fading models with low and high $L_{\text {peak,min }}$, to the measurement of the total CXRB from Moretti et al. (2003). This contribution is plotted as a function of the minimum detectable flux for sources with redshifts above $z=0.01,2,4,, 6,8$ and 10. We note that the resolved fraction is sensitive to uncertainties in the absolute value of the CXRB. We restrict our analysis of the resolved fraction to energies below $10 \mathrm{keV}$, where variations in the normalisation between experiments differ by $\sim 10$ per cent (see, e.g. Moretti et al. 2003).

In the $2-10 \mathrm{keV}$ band, we find that all models are con-

\begin{tabular}{|c|c|c|c|}
\hline$f_{\min }\left(\mathrm{erg} / \mathrm{s} / \mathrm{cm}^{2}\right)$ & Fading law & $z_{\min }$ & $x_{\mathrm{obs}}$ \\
\hline $3 . e-16$ & $\begin{array}{l}\text { Slow } \\
\text { Rapid } \\
L_{\text {peak,min }}=10^{11} \mathrm{~L}_{\odot} \\
\text { Rapid } \\
L_{\text {peak,min }}=10^{9} \mathrm{~L} \odot\end{array}$ & $\begin{array}{l}4 \\
6 \\
8 \\
4 \\
6 \\
8 \\
4 \\
6 \\
8\end{array}$ & $\begin{array}{l}3.1 \times 10^{-2} \\
1.9 \times 10^{-3} \\
5.9 \times 10^{-5} \\
6.0 \times 10^{-2} \\
4.2 \times 10^{-3} \\
1.3 \times 10^{-4} \\
6.0 \times 10^{-2} \\
4.2 \times 10^{-3} \\
1.3 \times 10^{-4}\end{array}$ \\
\hline $3 . e-17$ & $\begin{array}{l}\text { Slow } \\
\text { Rapid } \\
L_{\text {peak,min }}=10^{11} \mathrm{~L} \odot \\
\text { Rapid } \\
L_{\text {peak,min }}=10^{9} \mathrm{~L} \odot_{\odot}\end{array}$ & $\begin{array}{l}4 \\
6 \\
8 \\
4 \\
6 \\
8 \\
4 \\
6 \\
8\end{array}$ & $\begin{array}{l}1.3 \times 10^{-1} \\
1.8 \times 10^{-2} \\
1.6 \times 10^{-3} \\
2.1 \times 10^{-1} \\
4.8 \times 10^{-2} \\
5.5 \times 10^{-3} \\
2.0 \times 10^{-1} \\
4.0 \times 10^{-2} \\
4.5 \times 10^{-3}\end{array}$ \\
\hline $3 . e-18$ & $\begin{array}{l}\text { Slow } \\
\text { Rapid } \\
L_{\text {peak,min }}=10^{11} \mathrm{~L} \odot_{\odot} \\
\text { Rapid } \\
L_{\text {peak,min }}=10^{9} \mathrm{~L}_{\odot}\end{array}$ & $\begin{array}{l}4 \\
6 \\
8 \\
4 \\
6 \\
8 \\
4 \\
6 \\
8\end{array}$ & $\begin{array}{l}2.3 \times 10^{-1} \\
4.8 \times 10^{-2} \\
8.2 \times 10^{-3} \\
3.2 \times 10^{-1} \\
1.2 \times 10^{-1} \\
3.0 \times 10^{-2} \\
4.6 \times 10^{-1} \\
1.7 \times 10^{-1} \\
4.3 \times 10^{-2}\end{array}$ \\
\hline
\end{tabular}

Table 1. Table of the fraction of sources with observed $0.5-2 \mathrm{keV}$ fluxes above $f_{\min }$ and redshift above $z_{\min }$ for the 3 merger-driven models considered.

sistent with the total measured CXRB. Our models predict that $\sim 70-78$ per cent of the total $2-10 \mathrm{keV}$ CXRB is due to AGN with fluxes above the best current sensitivity level in this band $\left(\sim 1.4 \times 10^{-16} \mathrm{cgs}\right)$. This result is consistent with the $\sim 80$ per cent resolved fraction measured by Worsley et al. (2005) and Hickox \& Markevitch (2006). Our models remain consistent with the total CXRB at lower flux limits. At a sensitivity of $3 \times 10^{-18} \mathrm{cgs}$ - a sensitivity within reach of next generation instruments (see Section 5.3) - the resolved fraction increases to $\sim 87$ per cent depending on the model. Our models thus still leave room for the very hard spectrum sources need to make up the CXRB at energies $>8 \mathrm{keV}$ (Worslev et al. 2005) and/or the population of star-forming galaxies expected to contribute significantly at low flux levels (e.g. Bauer et al. 2004). We note that our calculated resolved fractions also depend on the analytic fits we have used below $z=2$. Using the more recent LDDE model from Silverman et al. (2007) we obtain lower resolved fractions $(\sim 62-75$ per cent due to sources above the current detection level), consistent with the more stringent optical selection criteria for this sample.

In the soft X-ray band $(0.5-2 \mathrm{keV})$, the fraction of the total CXRB flux due to sources above the current sensitivity level $\left(\sim 2.5 \times 10^{-17} \mathrm{cgs}\right)$ in our models is again consistent with the 80-90 per cent found by Worslev et al. (2005) and Hickox \& Markevitch (2006). However, at fainter flux levels the slow fading model overpredicts the total CXRB, again reflecting the excess of faint sources at $z \sim 2$ compared to the observed QLF. This contribution again depends on the faint 

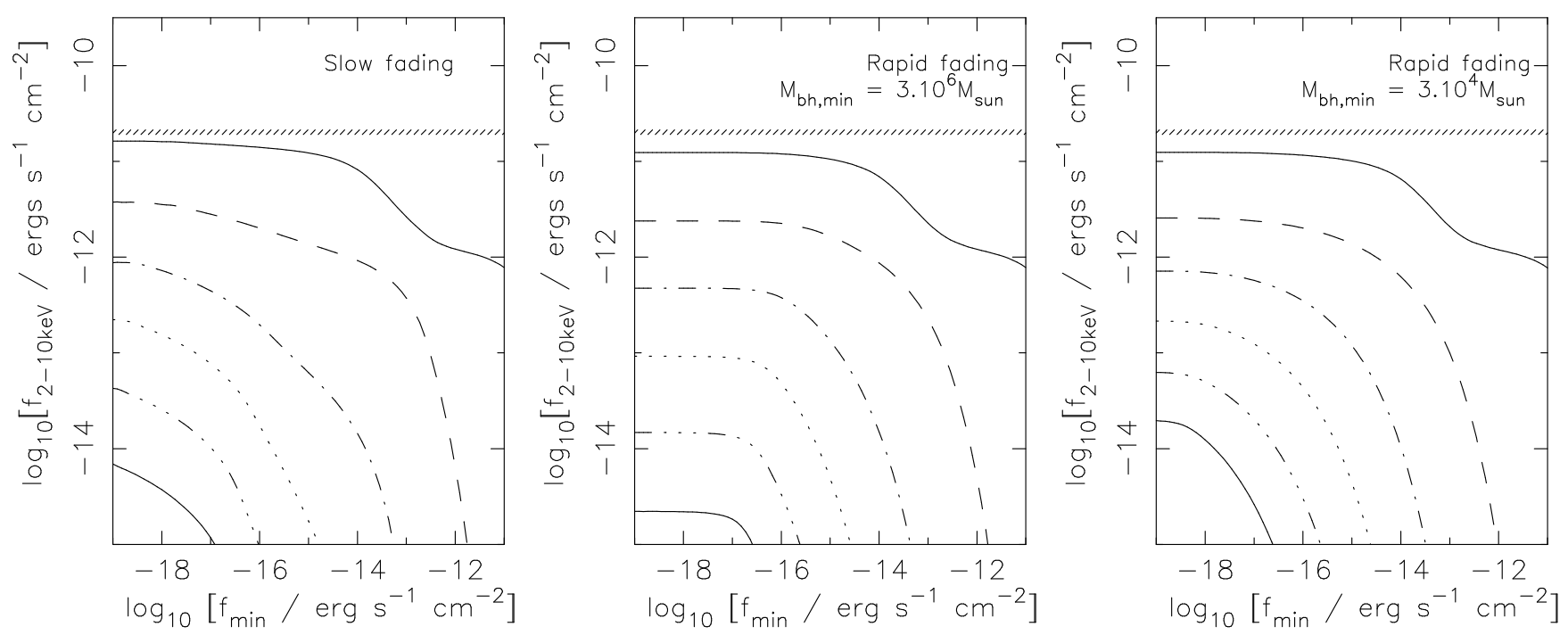

Figure 7. Integrated hard $(2-10 \mathrm{keV}) \mathrm{X}$-ray flux due to AGN for the models in Figure 5 . The hatched region shows the estimate of the total extragalactic CXRB in the $2-10 \mathrm{keV}$ band from Moretti et al. (2003). The left panel is for slow fading with $L_{\text {peak,min }}=10^{11} \mathrm{~L}_{\odot}$. The middle (right) panels show the results for rapid fading with $L_{\text {peak,min }}=10^{11} \mathrm{~L}_{\odot}\left(L_{\text {peak,min }}=10^{9} \mathrm{~L}_{\odot}\right)$
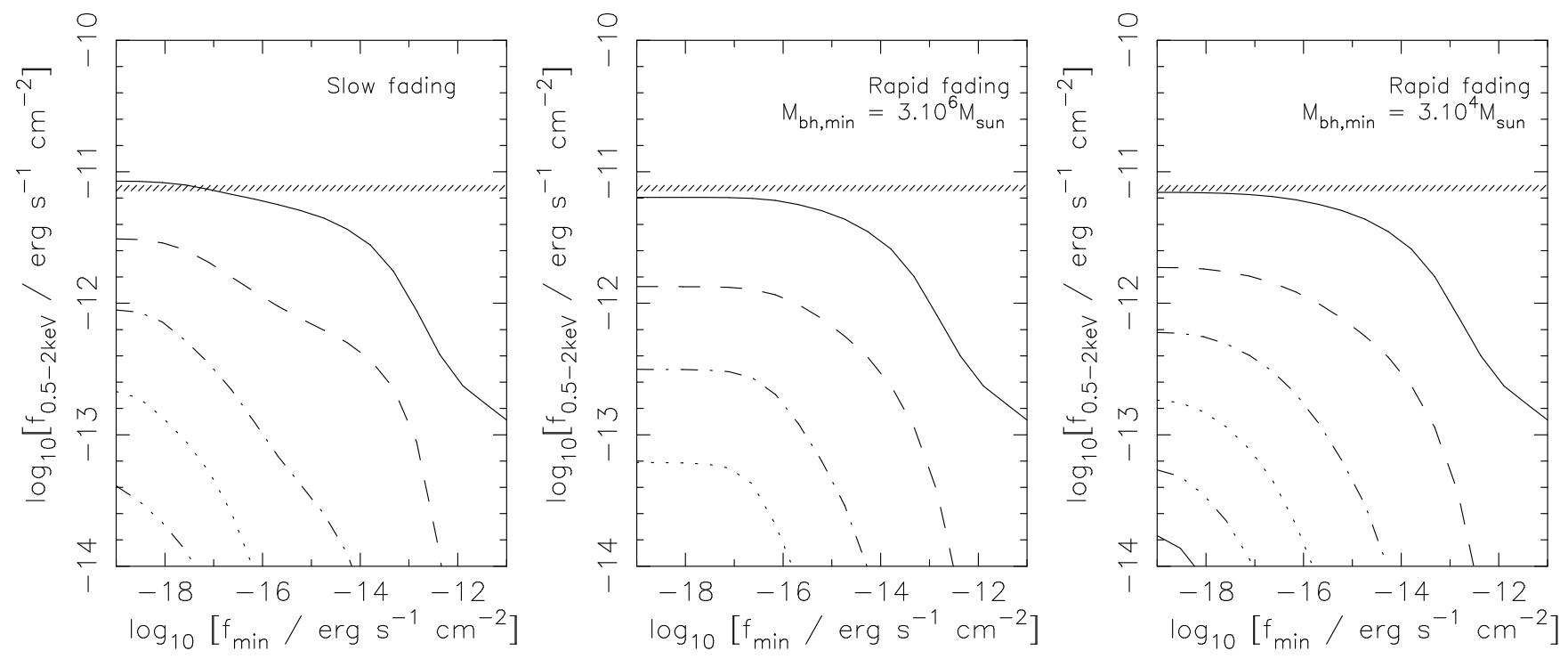

Figure 8. Integrated soft $(0.5-2 \mathrm{keV}) \mathrm{X}$-ray flux due to AGN for the models in Figure 6 . The hatched region shows the estimate of the total extragalactic component of the CXRB in the $0.5-2 \mathrm{keV}$ band from Moretti et al. (2003).

end behaviour of the fits to the data that we have adopted below $z \sim 2$. As mentioned, for the soft X-ray band we have chosen to use the LDDE2 model from Mivaii et al. (2001) which is constructed to reproduce $\sim 90$ of the total soft $\mathrm{X}$-ray background when integrated out to $z \sim 5$. Alternate faint end extrapolations may somewhat ease this excess. Using the Hasinger et al. (2005) LDDE fit to the LF of type-1 AGN below $z=2$ we find that the slow fading model saturates, but does not overpredict, the soft X-ray background. However the Hasinger et al. (2005) LDDE fit to the soft Xray type-1 QLF only accounts for $\sim 35$ per cent of the soft band CXRB, reflecting the fact that the sample of type-1 AGN used in Hasinger et al. (2005) account for only around 30 per cent of sources at faint and bright flux levels.
The resolved fractions are naturally lower for the slow and rapid fading models with $L_{\text {peak,min }}=10^{12} \mathrm{~L}_{\odot}($ chosen to reproduce the soft X-ray QLF at $z \sim 2$ ), and with this choice the slow fading model remains consistent with the total CXRB.

Recently, (Worsley et al. 2005) and Hickox \& Markevitch (2006) have pushed the limit for the unresolved background further by taking into account the stacked emission from galaxies detected with $H S T$ and $I R A C$. Indeed we find that if we integrate the flux due to sources below the current detection level and compare this to the measurement of the unresolved component as derived by Hickox \& Markevitch (2006), the soft and hard X-ray components are both too large for the slow fading 

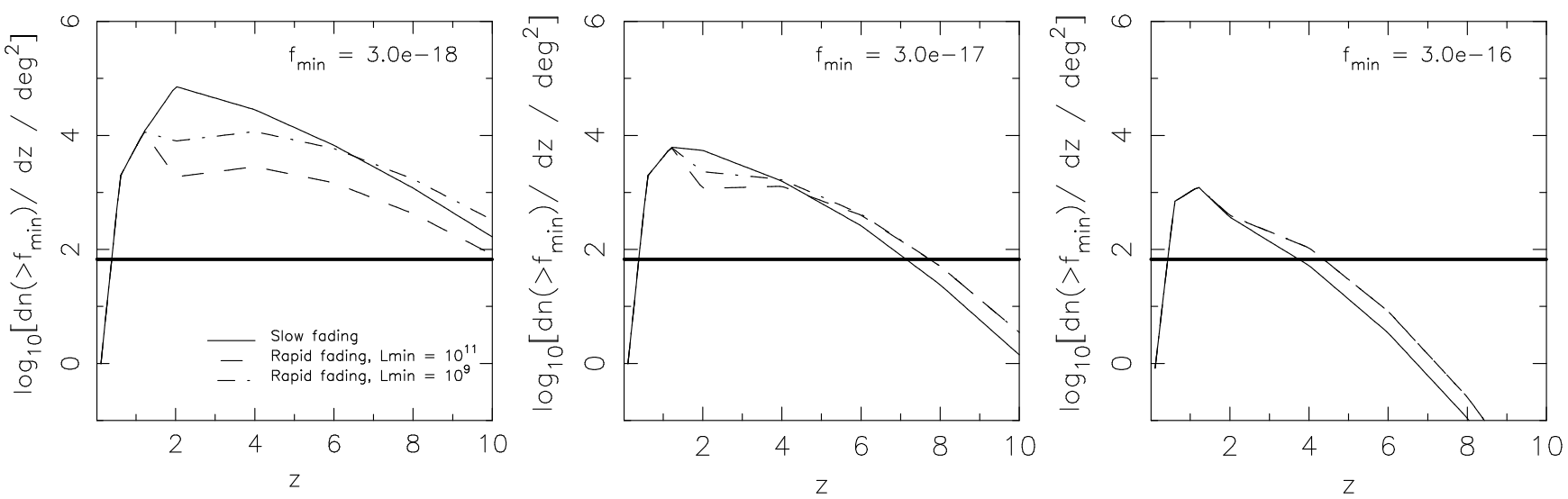

Figure 9. Redshift distribution of predicted counts in the $0.5-2 \mathrm{keV}$ band for each model; slow fading (solid line), rapid fading with $L_{\text {peak,min }}=10^{11} \mathrm{~L}_{\odot}$ (dashed line) and $L_{\text {peak,min }}=10^{9} \mathrm{~L} \odot$ (dot-dashed line) for three flux limits as labelled in cgs units. The horizontal line corresponds to a density of one source per XEUS WFI FOV.
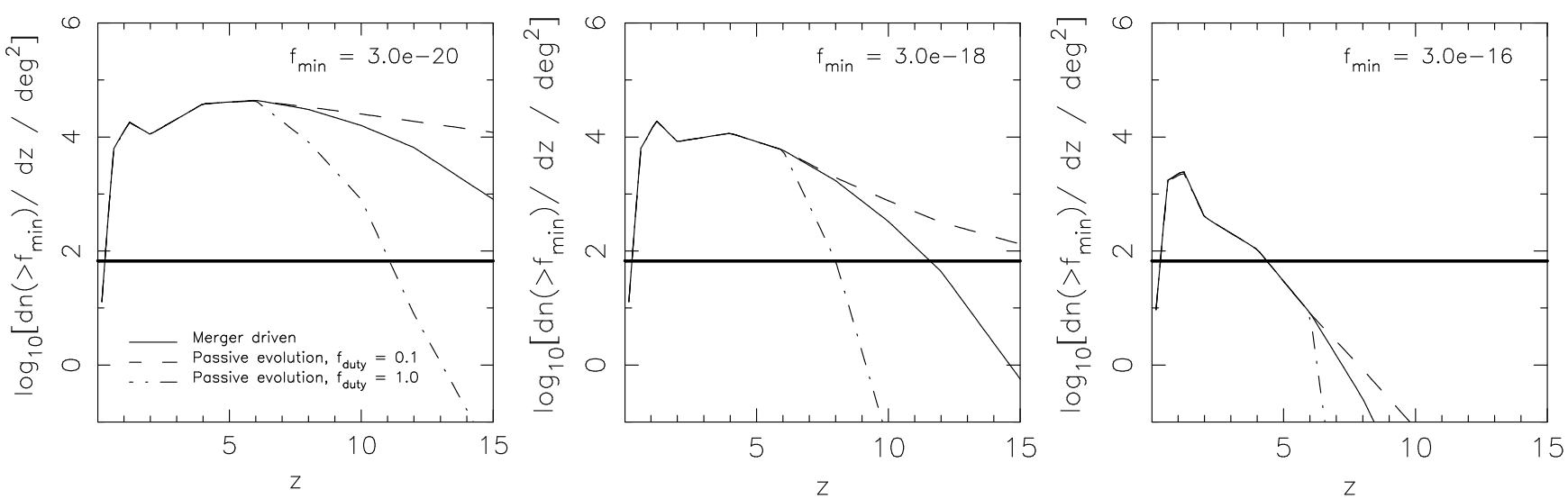

Figure 10. Redshift distribution of counts in the $0.5-2 \mathrm{keV}$ band for the rapid-fading merger driven model and passive evolution models as described in text; Merger-driven (solid line), passive evolution with total duty cycle $f_{\text {duty }}=0.1$ (dashed line) and $f_{\text {duty }}=1.0$ (dotdashed line) for three flux limits as labelled in cgs units. Note that the lowest flux limit is the anticipated sensitivity for the Generation-X mission, the middle for a 1 Ms observation with XEUS, and the right for a point-source within reach of current instruments.The horizontal line corresponds to a density of one source per XEUS WFI FOV $(7 \text { arcmin })^{2}$, which is similar in area to the proposed FOV for Generation-X.

model. Models like this for which sources with relatively flat spectra recover the entire unresolved CXRB in the soft and hard bands are likely to be in conflict with the overall measured shape of the CXRB above $8 \mathrm{keV}$ (e.g. Worslev et al. 2005; Comastri et al. 1995; Gilli et al. 2007). This consistency check argues further against the slow fading model as we have applied it, but we note that a flattening of the fading law slope for low mass black holes would alleviate the inconsistencies with the CXRB and the soft X-ray source counts. In particular the slow fading model with $L_{\text {peak,min }}=10^{12} \mathrm{~L}_{\odot}$ saturates, but does not over-predict, the unresolved component of the soft CXRB.

In a recent paper, Salvaterra et al. (2007) predict the contribution of high redshift AGN to the unresolved CXRB for a merger tree based model for the Eddington limited growth of $\mathrm{BH}$ seeds. They find that $\sim 5$ per cent of the unresolved 2-10 keV CXRB will be due to sources at $z>6$. This is similar to the $\sim 10$ per cent that we predict for the rapid fading model with $M_{\mathrm{bh} \text {,min }}=3 \times 10^{4} \mathrm{M}_{\odot}$, but significantly larger than we would predict for models with larger $M_{\mathrm{bh}, \min }$.

In the next section we focus on the predictions for the rapid fading models, since these are consistent with all available data, but have included the predictions for the slow fading model for reference.

\subsection{Future hard X-ray surveys and the early growth of supermassive black holes}

The sensitivities of the deepest X-ray surveys to date are photon limited and therefore independent of the background intensity. This may not be the case for the effective collecting area(s) and angular resolution(s) anticipated for XEUS and Constellation-X. Estimates of the point-source sensitivity for future instruments therefore potentially become dependent on the assumed unresolved extragalactic component (due to 
quasars and star-forming galaxies) and its flux distribution, as well as the effective collecting area and photon extraction radius (or resolution). Due to uncertainties in the decomposition of the X-ray background into contributions from the galaxy, star-forming galaxies and quasars, it is somewhat uncertain what the confusion limit of these telescopes will be. The projected point-source sensitivities for the next generation telescope peak for soft X-ray energies. At these energies the soft thermal galactic component is a significant portion of the background (see, e.g. Parmar et al. 1999), however the contribution of unresolved point sources may also play an important role if the ambitious goals for the resolution are not achieved. Since our models predict the contribution of quasars to this background, the expected sensitivity of an instrument like XEUS to high redshift sources may depend on the modelling of faint sources.

Hasinger et al. (2006) discuss the anticipated point source sensitivity of Constellation-X and XEUS. Assuming that the observations are confusion limited when there are fewer than 40 "beams" per source, combined with estimates of the background due to unresolved extragalactic sources, galactic emission and cosmic-rays, Hasinger et al. (2006) project that a $1 \mathrm{Ms}$ observation with XEUS will yield a point source sensitivity of $3 \times 10^{-18} \mathrm{erg} \mathrm{s}^{-1} \mathrm{~cm}^{-2}$, approximately 200 times better than that of XMM-Newton. This estimate presumes that XEUS's goal resolution of 2 arcseconds will be achieved. Achieving this resolution is expected to be extremely challenging, and the required resolution of 5 arcseconds is perhaps more realistic Hasinger et al. 2006). For this resolution the sensitivity degrades to $\sim$ $2 \times 10^{-17} \mathrm{erg} \mathrm{s}^{-1} \mathrm{~cm}^{-2}$ for a 1 Ms integration. Similarly, for Constellation-X Hasinger et al. (2006) project a point source sensitivity of $2 \times 10^{-17} \mathrm{erg} \mathrm{s}^{-1} \mathrm{~cm}^{-2}$ for a $1 \mathrm{Ms}$ observation for the Constellation-X goal resolution of 5 arcseconds.

Assuming a circular beam and applying the above confusion criterion suggests that XEUS will be able to identify point sources with densities below $\sim 8.6 \times 10^{4} \mathrm{deg}^{-2}$ if it reaches its goal resolution. Comparison with the source densities in our models suggests that XEUS should not suffer from confusion due to faint AGN at this flux limit for the rapid fading models, but would do so below $10^{-17} \mathrm{erg} \mathrm{s}^{-1} \mathrm{~cm}^{-2}$ for the slow fading model. The reduced sensitivity is not merely attributable to the excess in the predicted number of faint sources at $z \sim 2$, since the density of sources above $z \sim 4$ alone are sufficient to stop XEUS from reaching its projected sensitivity in this model. This suggests that the confusion limit for XEUS may be somewhat sensitive to the way quasars fade at $2<z<6$. However, we note that the same slow fading model with a higher minimum peak luminosity $L_{\text {peak,min }}=10^{12} \mathrm{~L}_{\odot}$ would not predict a degradation of the estimated point source sensitivity due to source confusion by faint AGN. Obviously the situation would be less favourable if the resolution goal of 2 arcseconds HEW could not be achieved. If the resolution is reduced to the required value of 5 arcminutes, we find the density of sources above $\sim 5 \times 10^{17} \mathrm{erg} \mathrm{s}^{-1} \mathrm{~cm}^{-2}$ exceeds the density defined by the confusion limit $\left(\sim 1.4 \times 10^{4} \mathrm{deg}^{-2}\right)$ for the rapid fading model with $L_{\text {peak,min }}=10^{9} \mathrm{~L} \odot$, and even the rather flat low-flux source density in the rapid fading model with $L_{\text {peak,min }}=10^{11}$ would limit the sensitivity to sources with fluxes above above $\sim 3 \times 10^{17} \mathrm{erg} \mathrm{s}^{-1} \mathrm{~cm}^{-2}$.
Similar sensitivities would be applicable to Constellation-X if it reaches its goal resolution.

The planned WF 4 is designed to have a field of view (FOV) of $(7 \text { arcminutes })^{2}$ [compared to $(5 \text { arcminutes })^{2}$ for Constellation-X, Garcia (2007)]. An unclustered distribution of sources with density $\sim 6.7 \times 10^{1} \mathrm{deg}^{-2}$ will then contain one source per WFI FOV. We highlight this density as a horizontal line in Figures 9 and 10 for reference. For the rapid-fading models, for which observations should not be confusion limited down to the goal sensitivity of XEUS, we predict significant numbers of sources (more than one per WFI FOV) out to $z \sim 10$. To put these sensitivities in context for models of SMBH growth, in Figure 11 we plot the $0.5-2 \mathrm{keV}$ flux in the observed frame for BHs accreting at their Eddington limit (using the Hopkins et al. (2007) $z$-independent SED) as a function of the source redshift. The lowest mass BH that may be seen accreting at its Eddington limit at $z \sim 6-10$ for a 1 Ms observation with XEUS is in the range $\sim 10^{5}-5 \times 10^{5} \mathrm{M}_{\odot}$. For a model in which only BHs larger than $\sim 3 \times 10^{7} \mathrm{M}_{\odot}$ are active (i.e. $L_{\text {peak,min }}=10^{12} \mathrm{~L}_{\text {sun }}$ ), the redshift limit up to which we predict more than one source per XEUS field is reduced to 8 (7) for the slow (rapid) fading model.

Contrastingly a telescope with 5 arcsecond resolution, which as mentioned above may be confusion limited below $\sim 3 \times 10^{-17} \mathrm{erg} \mathrm{s}^{-1} \mathrm{~cm}^{-2}$, may be sensitive enough to detect to more than one source per XEUS (Constellation-X) FOV up to $z \sim 7(z \sim 6)$, but detection of significant numbers of sources at higher redshifts would require surveying many XEUS/Constellation-X fields (see Figure 9). This result changes little for the case $L_{\text {peak,min }}=10^{12} \mathrm{~L}_{\odot}$, with approximately one source per XEUS field out to $z \sim 6$ predicted independently of the fading law.

The confusion limits we have discussed represent the best case for each model given the assumptions in Hasinger et al. (2006) for the contribution from galactic emission and star-forming galaxies, and the true confusion limits may therefore be worse if these have been underestimated.

Improvements in the resolution of hard X-ray telescopes will naturally improve the confusion limit for the point source sensitivity (where it is limited by discrete sources), particularly for the case of rapid fading in which the source counts rise slowly with decreasing flux. NASA's mission concept Generation-X has a goal resolution of 0.1 arcseconds and $100 \mathrm{~m}^{2}$ of collecting area yielding a photometric sensitivity of $\sim 2.2 \times 10^{-20} \mathrm{ergs} \mathrm{s}^{-1} \mathrm{~cm}^{-2}$ at $0.1-10 \mathrm{keV}^{5}$. This resolution would certainly avoid confusion due to faint quasars, even for the slow fading model where there are many faint foreground sources confusing the detection of sources at $z \gtrsim 5$.

With the proposed sensitivity of Generation-X, unobscured BHs more massive than around a few $\times 1000 \mathrm{M}_{\odot}$ would be detectable out to redshifts approaching 15 (see Figure 11). Detection of the seeds of SMBHs is one of the prime science goals of Generation-X. The number of detectable objects at $z \sim 6-15$ will obviously depend on the masses of

\footnotetext{
4 ftp://ftp.xray.mpe.mpg.de/people/bol/xeus/XEUS_150108.pdf

5 http://www.psu.edu/dept/csrp/missions_awarded_genx.htm
} 
seed BHs and the growth history of BHs at very high redshifts.

In Figure 10 we compare the predicted number of detectable sources for the rapid fading merger-driven model (solid line) to the passive evolution models with $f_{\text {duty }}=0.1$ (dotted line) and $f_{\text {duty }}=1.0$ (dot-dashed line) for three values of the point-source sensitivity. The left panel shows the evolution in the density of sources with fluxes above the sensitivity goal of Generation-X. The space density of observable sources at $z \sim 15$ depends very sensitively on the model assumed. The FOV of Generation-X is expected to be at least $(5 \text { arcminutes })^{2}$, with fields as big as $(15 \text { arcminutes })^{2}$ considered in the design study (Windhorst et al. 2006). Here we assume the FOV of Generation-X is the same as for XEUS for ease of comparison. For the merger driven model, with a minimum active $\mathrm{BH}$ mass of $\sim 3 \times 10^{4} \mathrm{M}_{\odot}$ we predict significant numbers of sources, still around 10 per FOV, out to $z=15$. The situation is even more promising if the BHs are evolving passively with a small duty cycle, with up to $\sim 100$ sources per field up to $z=15$. If the BHs are evolving rapidly there is still more than one source per field out to $z \sim 11$, but the density drops very rapidly with increasing redshift for a scenario in which all $\mathrm{BHs}$ are growing via accretion at their Eddington limit. At the goal sensitivity of XEUS, the maximum redshift at which there is one source predicted per FOV also varies widely; ranging from $z \sim 8$ for continuous growth to $z \sim 15$ for slowly growing BHs.

\section{SUMMARY AND CONCLUSIONS}

We have presented a hybrid model for the redshift evolution of the X-ray emission connected with the fuelling of supermassive black holes with the aim of assessing the prospects of detecting quasars via their X-ray emission at $z>6$. At $z \lesssim 2$ we have used the observed X-ray luminosity functions, at $2<z<6$ we have adopted a CDM merger-driven model for the evolution of the emission from supermassive black holes combined with a slow and a rapid fading law and we have explored a range of assumptions for the growth of supermassive black holes at $z>6$.

For appropriate choices for the efficiency of black hole formation in a dark matter halo and the characteristic quasar lifetime, our model is in good agreement with the observed optical and soft and hard X-ray quasar luminosity functions at $0<z<6$ for both the slow and the rapid fading law. The only disagreement that occurs is for the faint end of the X-ray luminosity functions where our merger model combined with the slow fading model suggested by Hopkins et al. (2005a) based on detailed numerical simulations predicts too many faint objects. It consequently also overpredicts the soft X-ray background suggesting that the slow fading model of Hopkins et al. (2005a) cannot extend to black hole masses much below $10^{7} \mathrm{M}_{\odot}$. With a more rapid fading law our models are well within the limits of the integrated CXRB, leaving room for the population of star-forming galaxies expected at low flux densities, and the population of Compton-thick sources needed to explain the overall spectral shape of the CXRB.

The main aim of our study is to assess the prospects of planned and proposed X-ray missions like Constellation$\mathrm{X}$, XEUS and Generation-X to study the build-up of black holes at $z>6$. With a point source sensitivity of $3 \times$ $10^{-17} \mathrm{ergs} \mathrm{s}^{-1} \mathrm{~cm}^{-2}$ for a $1 \mathrm{Ms}$ exposure, Constellation$\mathrm{X}$ will detect significant numbers of black holes at $z \sim 5-6$ but will probably not yet reach the necessary sensitivity to detect significant numbers of sources at $z>6$. This conclusion holds independently of our assumptions for the growth of BHs at $z>6$. For XEUS with its anticipated ten times superior point source sensitivity, the prospects for detecting sources at $z>6$ is much more favourable. Up to 17 per cent of the approximately 100 sources in the 49 square arcminute FOV expected for the rapid fading model with a minimum active black hole mass of $\sim 3 \times 10^{4} \mathrm{M}_{\odot}$ are expected to be at $z>6$. If our merger driven model can be extrapolated to $z>6$ or if black holes grew with a duty cycle of about 10 per cent, XEUS would detect significant numbers of black holes with a rather flat redshift distribution out to $z \sim 10$. In either case, observable X-ray emission would have to accompany the growth of supermassive black holes starting from seed black holes of $\gtrsim 10^{4} \mathrm{M}_{\odot}$ or smaller. For the more remote prospect of Generation-X, with an anticipated point source sensitivity yet a factor 100 better again, the flat redshift distribution could extent to $z \sim 15$ and beyond.

If black holes grew much faster at $z>6$ as in our Eddington limited growth model with a duty fraction of unity, which would be required for Eddington limited growth of the most massive black holes from stellar mass seed black holes, neither XEUS nor Generation-X would detect many black holes at $z \gtrsim 10$. However, while such rapid growth may occur and may indeed be necessary for the most massive black holes at $z \sim 6$, it appears unlikely that this will be the norm for the majority of black holes. The prospects that XEUS (and Generation-X) will unravel the growth history of black holes at $z>6$ and beyond are thus excellent.

\section{ACKNOWLEDGMENTS}

KJR was supported by an Overseas Research Scholarship, the Cambridge Australia Trust, the School of Physics, University of Melbourne and an EARA visiting post-graduate scholarship hosted by MPA during the course of this work. We would like to thank the referee for a detailed and helpful report.

\section{REFERENCES}

Aird J., Nandra K., Georgakakis A., Laird E. S., Steidel C. C., Sharon C., 2008, ArXiv e-prints, 804.0760

Archibald E. N., Dunlop J. S., Jimenez R., Friaça A. C. S., McLure R. J., Hughes D. H., 2002, MNRAS, 336, 353

Babić A., Miller L., Jarvis M. J., Turner T. J., Alexander

D. M., Croom S. M., 2007, A\&A, 474, 755

Barger A. J., Cowie L. L., Mushotzky R. F., Yang Y., Wang W.-H., Steffen A. T., Capak P., 2005, AJ, 129, 578

Barkana R., Loeb A., 2001, Phys. Rep., 349, 125

Bauer F. E., Alexander D. M., Brandt W. N., Schneider D. P., Treister E., Hornschemeier A. E., Garmire G. P., 2004, AJ, 128, 2048

Best P. N., von der Linden A., Kauffmann G., Heckman

T. M., Kaiser C. R., 2007, MNRAS, 379, 894 
Detecting high redshift quasars in $X$-rays

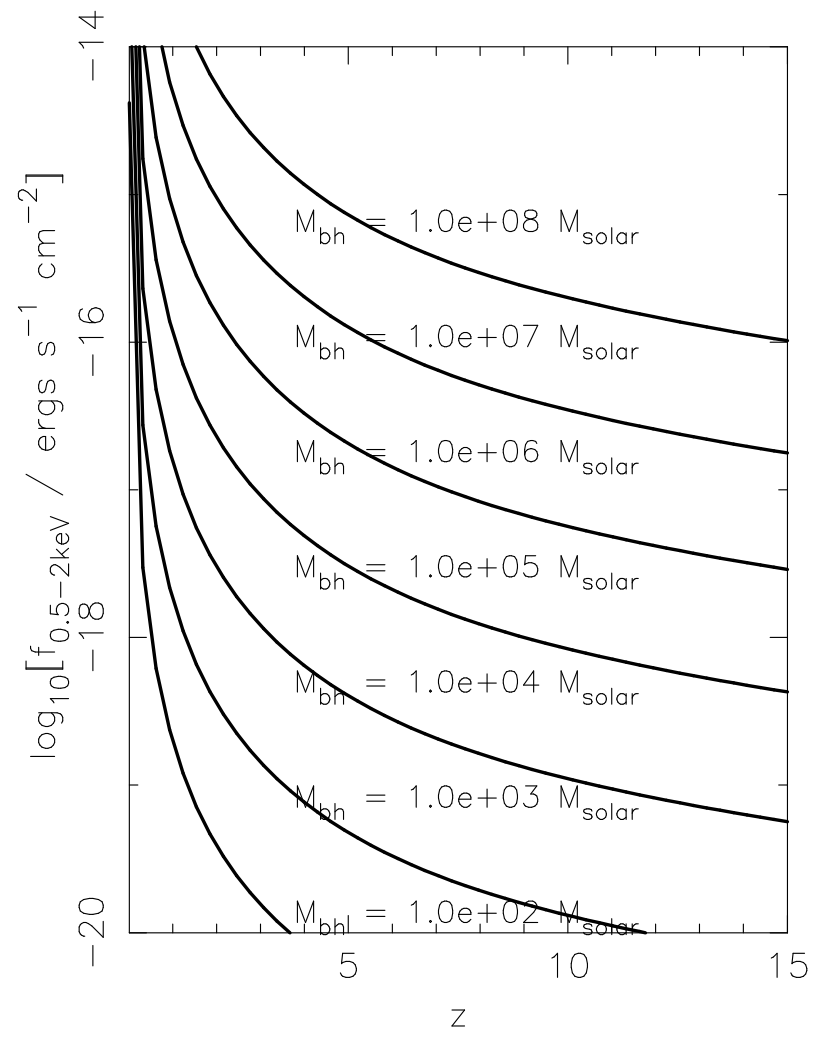

Figure 11. Observed $0.5-2 \mathrm{keV}$ flux from a $\mathrm{BH}$ with the Hopkins et al. (2007) SED shining at its Eddington limit as a function of source redshift. BH masses as labelled.

Bongiorno A., Zamorani G., Gavignaud I., Marano B., Paltani S., Mathez G., Møller P., Picat J. P., Cirasuolo M., Lamareille F., Bottini D., Garilli B., Le Brun V., Le Fèvre O., Maccagni D., Scaramella R., Scodeggio M., Tresse L., Vettolani G., Zanichelli A., Adami C., Arnouts S., Bardelli S., Bolzonella M., Cappi A., Charlot S., Ciliegi P., Contini T., Foucaud S., Franzetti P., Guzzo L., Ilbert O., Iovino A., McCracken H. J., Marinoni C., Mazure A., Meneux B., Merighi R., Pellò R., Pollo A., Pozzetti L., Radovich M., Zucca E., Hatziminaoglou E., Polletta M., Bondi M., Brinchmann J., Cucciati O., de La Torre S., Gregorini L., Mellier Y., Merluzzi P., Temporin S., Vergani D., Walcher C. J., 2007, A\&A, 472, 443

Boyle B. J., Shanks T., Croom S. M., Smith R. J., Miller L., Loaring N., Heymans C., 2000, MNRAS, 317, 1014

Bromley J. M., Somerville R. S., Fabian A. C., 2004, MNRAS, 350, 456

Cattaneo A., Blaizot J., Weinberg D. H., Kereš D., Colombi S., Davé R., Devriendt J., Guiderdoni B., Katz N., 2007, MNRAS, 377, 63

Comastri A., Setti G., Zamorani G., Hasinger G., 1995, A\&A, 296, 1

Cowie L. L., Garmire G. P., Bautz M. W., Barger A. J., Brandt W. N., Hornschemeier A. E., 2002, ApJ, 566, L5

Croom S. M., Smith R. J., Boyle B. J., Shanks T., Miller L., Outram P. J., Loaring N. S., 2004, MNRAS, 349, 1397 Croton D. J., Springel V., White S. D. M., De Lucia G., Frenk C. S., Gao L., Jenkins A., Kauffmann G., Navarro J. F., Yoshida N., 2006, MNRAS, 365, 11
Di Matteo T., Springel V., Hernquist L., 2005, Nature, 433, 604

Dunn R. J. H., Fabian A. C., 2006, MNRAS, 373, 959

Fan X., Hennawi J. F., Richards G. T., Strauss M. A., Schneider D. P., Donley J. L., Young J. E., Annis J., Lin H., Lampeitl H., Lupton R. H., Gunn J. E., Knapp G. R., Brandt W. N., Anderson S., Bahcall N. A., Brinkmann J., Brunner R. J., Fukugita M., Szalay A. S., Szokoly G. P., York D. G., 2004, AJ, 128, 515

Fan X., Strauss M. A., Schneider D. P., Becker R. H., White R. L., Haiman Z., Gregg M., Pentericci L., Grebel E. K., Narayanan V. K., Loh Y.-S., Richards G. T., Gunn J. E., Lupton R. H., Knapp G. R., Ivezić Ž., Brandt W. N., Collinge M., Hao L., Harbeck D., Prada F., Schaye J., Strateva I., Zakamska N., Anderson S., Brinkmann J., Bahcall N. A., Lamb D. Q., Okamura S., Szalay A., York D. G., 2003, AJ, 125, 1649

Ferrarese L., 2002, ApJ, 578, 90

Ferrarese L., Merritt D., 2000, ApJ, 539, L9

Fiore F., Brusa M., Cocchia F., Baldi A., Carangelo N., Ciliegi P., Comastri A., La Franca F., Maiolino R., Matt G., Molendi S., Mignoli M., Perola G. C., Severgnini P., Vignali C., 2003, A\&A, 409, 79

Fontanot F., Cristiani S., Monaco P., Nonino M., Vanzella E., Brandt W. N., Grazian A., Mao J., 2007, A\&A, 461, 39

Fontanot F., Monaco P., Cristiani S., Tozzi P., 2006, MNRAS, 373, 1173

Garcia M. R., 2007, in American Astronomical Society Meeting Abstracts, Vol. 211, American Astronomical Society Meeting Abstracts, p. 98.07

Gaskell C. M., Goosmann R. W., Antonucci R. R. J., Whysong D. H., 2004, ApJ, 616, 147

Gebhardt K., Kormendy J., Ho L. C., Bender R., Bower G., Dressler A., Faber S. M., Filippenko A. V., Green R., Grillmair C., Lauer T. R., Magorrian J., Pinkney J., Richstone D., Tremaine S., 2000, ApJ, 543, L5

Gilli R., Comastri A., Hasinger G., 2007, A\&A, 463, 79

Greene J. E., Ho L. C., 2007a, ArXiv e-prints, 707

-, 2007b, ApJ, 667, 131

Haehnelt M. G., 2004, in Coevolution of Black Holes and

Galaxies, Ho L. C., ed., pp. 405--

Haehnelt M. G., Natarajan P., Rees M. J., 1998, MNRAS, 300,817

Haiman Z., Loeb A., 1999, ApJ, 521, L9

Hasinger G., Arnaud M., Barcons X., Barret D., Bleeker J. A., Böhringer H., Comastri A., Fabian A. C., Kaastra J. S., Kunieda H., Mendez M., Parmar A. N., Palumbo G. G. C., Takahashi T., Turner M. J. L., 2006, in Presented at the Society of Photo-Optical Instrumentation Engineers (SPIE) Conference, Vol. 6266, Space Telescopes and Instrumentation II: Ultraviolet to Gamma Ray. Edited by Turner, Martin J. L.; Hasinger, Günther. Proceedings of the SPIE, Volume 6266, pp. 62661N (2006)., Turner M. J. L., Hasinger G., eds.

Hasinger G., Miyaji T., Schmidt M., 2005, A\&A, 441, 417 Hickox R. C., Markevitch M., 2006, ApJ, 645, 95

Hopkins P. F., Hernquist L., Cox T. J., Di Matteo T., Martini P., Robertson B., Springel V., 2005a, ApJ, 630, 705

Hopkins P. F., Hernquist L., Cox T. J., Di Matteo T., Robertson B., Springel V., 2005b, ApJ, 632, 81

—, 2006, ApJS, 163, 1 
Hopkins P. F., Richards G. T., Hernquist L., 2007, ApJ, 654, 731

Hopkins P. F., Strauss M. A., Hall P. B., Richards G. T., Cooper A. S., Schneider D. P., Vanden Berk D. E., Jester S., Brinkmann J., Szokoly G. P., 2004, AJ, 128, 1112

Jiang L., Fan X., Annis J., Becker R. H., White R. L., Chiu K., Lin H., Lupton R. H., Richards G. T., Strauss M. A., Jester S., Schneider D. P., 2007, ArXiv e-prints, 708

Kauffmann G., Haehnelt M., 2000, MNRAS, 311, 576

Kauffmann G., Heckman T. M., Tremonti C., Brinchmann J., Charlot S., White S. D. M., Ridgway S. E., Brinkmann J., Fukugita M., Hall P. B., Ivezić Ž., Richards G. T., Schneider D. P., 2003, MNRAS, 346, 1055

Kollmeier J. A., Onken C. A., Kochanek C. S., Gould A., Weinberg D. H., Dietrich M., Cool R., Dey A., Eisenstein D. J., Jannuzi B. T., Le Floc'h E., Stern D., 2006, ApJ, 648, 128

Lacey C., Cole S., 1993, MNRAS, 262, 627

Li C., Kauffmann G., Heckman T. M., White S. D. M., Jing Y. P., 2007a, ArXiv e-prints, 712

Li Y., Hernquist L., Robertson B., Cox T. J., Hopkins P. F., Springel V., Gao L., Di Matteo T., Zentner A. R., Jenkins A., Yoshida N., 2007b, ApJ, 665, 187

Madau P., Rees M. J., 2001, ApJ, 551, L27

Maiolino R., Shemmer O., Imanishi M., Netzer H., Oliva E., Lutz D., Sturm E., 2007, A\&A, 468, 979

Malbon R. K., Baugh C. M., Frenk C. S., Lacey C. G., 2006, ArXiv Astrophysics e-prints

Martínez-Sansigre A., Rawlings S., Bonfield D. G., Mateos S., Simpson C., Watson M., Almaini O., Foucaud S., Sekiguchi K., Ueda Y., 2007, MNRAS, 379, L6

Marulli F., Branchini E., Moscardini L., Volonteri M., 2007, MNRAS, 375, 649

McLure R. J., Dunlop J. S., 2004, MNRAS, 352, 1390

Miyaji T., Griffiths R. E., 2002, ApJ, 564, L5

Miyaji T., Hasinger G., Schmidt M., 2000, A\&A, 353, 25

-, 2001, A\&A, 369, 49

Moretti A., Campana S., Lazzati D., Tagliaferri G., 2003, ApJ, 588, 696

Parmar A. N., Guainazzi M., Oosterbroek T., Orr A., Favata F., Lumb D., Malizia A., 1999, A\&A, 345, 611

Pei Y. C., 1992, ApJ, 395, 130

Press W. H., Schechter P., 1974, ApJ, 187, 425

Rhook K. J., Haehnelt M. G., 2006, MNRAS, 373, 623

Richards G. T., Strauss M. A., Fan X., Hall P. B., Jester S., Schneider D. P., Vanden Berk D. E., Stoughton C., Anderson S. F., Brunner R. J., Gray J., Gunn J. E., Ivezić Ž., Kirkland M. K., Knapp G. R., Loveday J., Meiksin A., Pope A., Szalay A. S., Thakar A. R., Yanny B., York D. G., Barentine J. C., Brewington H. J., Brinkmann J., Fukugita M., Harvanek M., Kent S. M., Kleinman S. J., Krzesiński J., Long D. C., Lupton R. H., Nash T., Neilsen Jr. E. H., Nitta A., Schlegel D. J., Snedden S. A., 2006, AJ, 131, 2766

Rines K., Diaferio A., Natarajan P., 2007, ApJ, 657, 183

Risaliti G., Maiolino R., Salvati M., 1999, ApJ, 522, 157

Salvaterra R., Haardt F., Volonteri M., 2007, MNRAS, 374, 761

Shankar F., Mathur S., 2007, ApJ, 660, 1051

Shankar F., Weinberg D. H., Miralda-Escude' J., 2007, ArXiv e-prints, 710

Sheth R. K., Tormen G., 1999, MNRAS, 308, 119
Shields G. A., Gebhardt K., Salviander S., Wills B. J., Xie B., Brotherton M. S., Yuan J., Dietrich M., 2003, ApJ, 583,124

Sijacki D., Springel V., 2006, MNRAS, 366, 397

Sijacki D., Springel V., di Matteo T., Hernquist L., 2007, MNRAS, 380, 877

Silk J., Rees M. J., 1998, A\&A, 331, L1

Silverman J. D., Green P. J., Barkhouse W. A., Kim D. ., Kim M., Wilkes B. J., Cameron R. A., Hasinger G., Jannuzi B. T., Smith M. G., Smith P. S., Tananbaum H., 2007, ArXiv e-prints, 710

Simpson C., 2005, MNRAS, 360, 565

Steffen A. T., Strateva I., Brandt W. N., Alexander D. M., Koekemoer A. M., Lehmer B. D., Schneider D. P., Vignali C., 2006, AJ, 131, 2826

Treister E., Urry C. M., Van Duyne J., Dickinson M., Chary R.-R., Alexander D. M., Bauer F., Natarajan P., Lira P., Grogin N. A., 2006, ApJ, 640, 603

Ueda Y., Akiyama M., Ohta K., Miyaji T., 2003, ApJ, 598, 886

Volonteri M., 2006, ArXiv Astrophysics e-prints

Volonteri M., Haardt F., Madau P., 2003, ApJ, 582, 559

Volonteri M., Lodato G., Natarajan P., 2007, ArXiv eprints, 709

Windhorst R. A., Cameron R. A., Brissenden R. J., Elvis M. S., Fabbiano G., Gorenstein P., Reid P. B., Schwartz D. A., Bautz M. W., Figueroa-Feliciano E., Petre R., White N. E., Zhang W. W., 2006, New Astronomy Review, 50, 121

Wolf C., Wisotzki L., Borch A., Dye S., Kleinheinrich M., Meisenheimer K., 2003, A\&A, 408, 499

Worsley M. A., Fabian A. C., Bauer F. E., Alexander D. M., Hasinger G., Mateos S., Brunner H., Brandt W. N., Schneider D. P., 2005, MNRAS, 357, 1281

Wyithe J. S. B., Loeb A., 2002, ApJ, 581, 886

—, 2003, ApJ, 595, 614 